\title{
A multi-omics approach to visualize early neuronal differentiation in 4D
}

Athina Samara ${ }^{\$, 1,2, \#}$, Mari Spildrejorde ${ }^{\$, 3,4,5}$, Ankush Sharma ${ }^{\$, 6,7,8,{ }^{*}}$, Martin Falck ${ }^{3,8, \&}$, Magnus Leithaug ${ }^{4, \S}$, Stefania Modafferi ${ }^{4,+}$, Pål Marius Bjørnstad ${ }^{4}$, Ganesh Acharya $^{9,10}$, Kristina Gervin ${ }^{3,11,12}$, Robert Lyle R,4,13,\# and Ragnhild Eskeland $^{3,7,14, \#}$

\$ Equal co-authorship contribution.

\# Co-corresponding authors

${ }^{1}$ Division of Clinical Paediatrics, Department of Women's and Children's Health, Karolinska Institutet, Sweden

${ }^{2}$ Astrid Lindgren Children's Hospital Karolinska University Hospital, Stockholm, Sweden

${ }^{3}$ PharmaTox Strategic Research Initiative, Faculty of Mathematics and Natural Sciences, University of Oslo, Norway

${ }^{4}$ Department of Medical Genetics, Oslo University Hospital and University of Oslo, Norway

${ }^{5}$ Institute of Clinical Medicine, Faculty of Medicine, University of Oslo, Oslo, Norway.

${ }^{6}$ Department of Informatics, University of Oslo, Norway

${ }^{7}$ Institute of Basic Medical Sciences, University of Oslo, Oslo, Norway

${ }^{8}$ Department of Biosciences, University of Oslo, Norway

${ }^{9}$ Division of Obstetrics and Gynecology, Department of Clinical Science, Intervention and Technology (CLINTEC), Karolinska Institutet, Alfred Nobels Allé 8, SE-14152, Stockholm, Sweden.

${ }^{10}$ Center for Fetal Medicine, Karolinska University Hospital Huddinge, SE-14186

Stockholm, Sweden.

${ }^{11}$ Pharmacoepidemiology and Drug Safety Research Group, Department of Pharmacy, School of Pharmacy, University of Oslo, Norway

${ }^{12}$ Division of Clinical Neuroscience, Department of Research and Innovation, Oslo University Hospital, Oslo, Norway

${ }^{13}$ Centre for Fertility and Health, Norwegian Institute of Public Health, Oslo, Norway

${ }^{14}$ Lead contact

\#Correspondence: athina.samara@ki.se (A.S.), robert.lyle@medisin.uio.no (R.L.), ragnhild.eskeland@medisin.uio.no (R.E.)

*Current address: Department of Cancer Immunology, Institute for Cancer Research, Oslo University Hospital, and KG Jebsen Centre for B-cell malignancies, Institute for Clinical Medicine, University of Oslo, Norway.

\& Current address: Department of Medical Genetics, Oslo University Hospital, and University of Oslo, Norway.

$\S$ Current address: Department of Analysis and Diagnostics, Section for Molecular Biology, Norwegian Veterinary Institute, Ås, Norway.

+ Current address: Istituto di Genetica Molecolare, CNR - Consiglio Nazionale delle Ricerche, Pavia, Italy. 


\section{Summary}

Neuronal differentiation of pluripotent stem cells is an established method to study physiology, disease and medication safety. However, the sequence of events in human neuronal differentiation and the ability of in vitro models to recapitulate early brain development are poorly understood. We developed a protocol optimized for the study of early human brain development and neuropharmacological applications. We comprehensively characterized gene expression and epigenetic profiles at four timepoints, as the cells differentiate from embryonic stem cells towards a heterogenous population of progenitors, immature and mature neurons bearing telencephalic signatures. A multi-omics roadmap of neuronal differentiation, combined with searchable interactive gene analysis tools, allows for extensive exploration of early neuronal development and the effect of medications.

\section{Graphical Abstract}

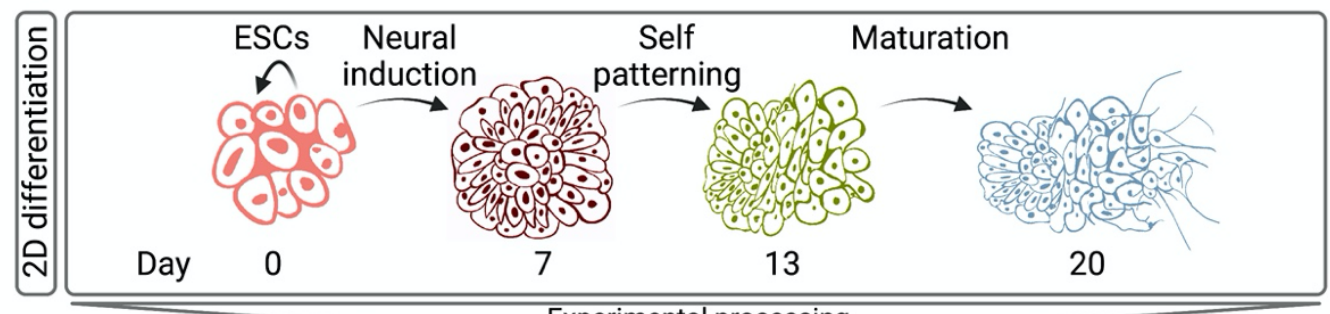

Experimental processing

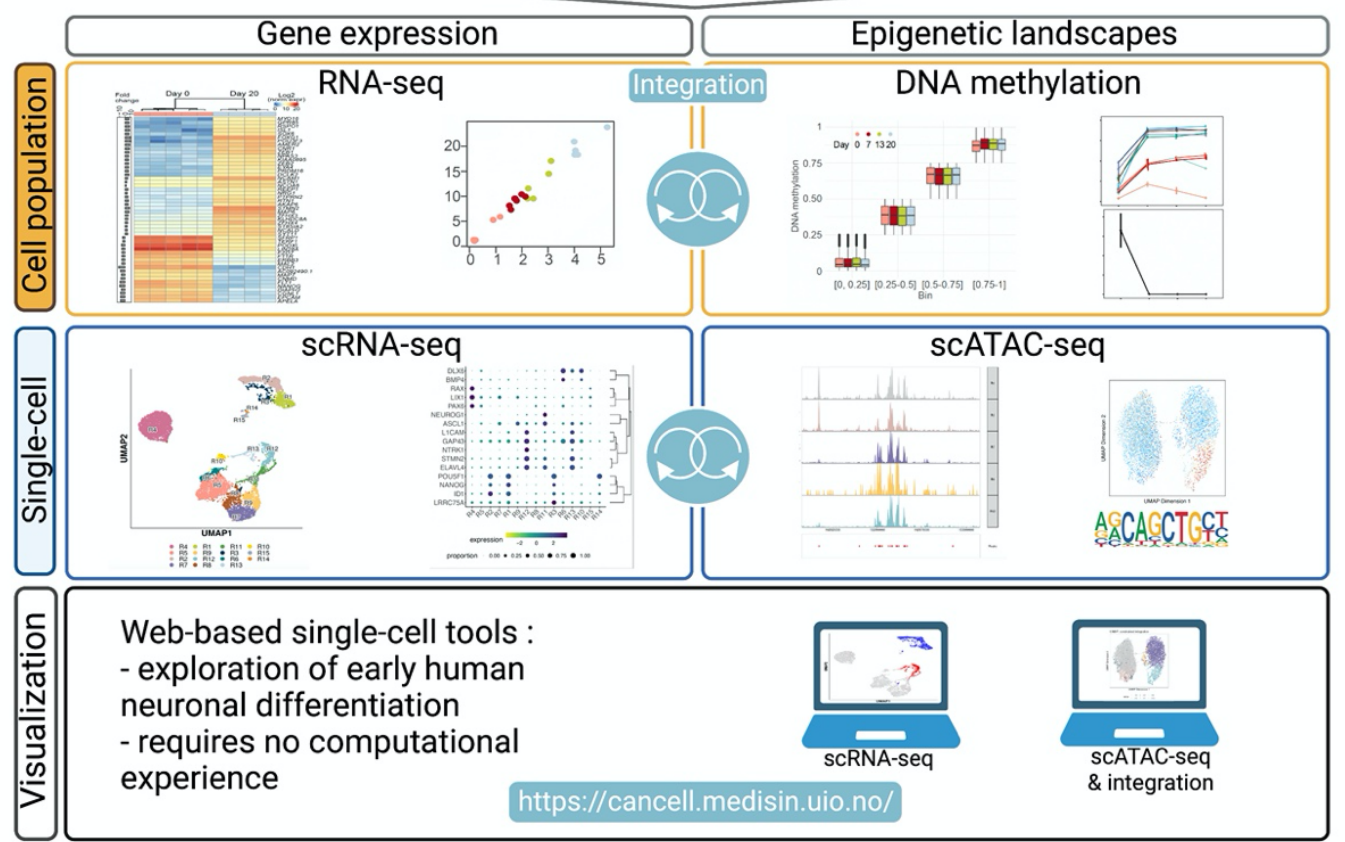




\section{Highlights}

- Multi-omics charting a new neuronal differentiation protocol for human ES cells

- $\quad$ Single-cell analyses reveals marker genes during neuronal differentiation

- Identified transcriptional waves similar to early human brain development

- $\quad$ Searchable tools to visualize single-cell gene expression and chromatin state

\section{In Brief}

We have developed a novel protocol for human embryonic stem cells to study neural induction and early neuronal differentiation. Multi-omics analyses uncovered cell populations, genes and transcriptional waves defining cell fate commitment. We comprehensively describe epigenetic landscapes and gene expression and provide searchable analysis tools for exploration of the data.

\section{Keywords}

Single-cell RNA-seq, scATAC-seq, human embryonic stem cells, neuronal differentiation, DNA methylation, telencephalic signatures 


\section{Introduction}

Neuronal differentiation of pluripotent stem cells (PSCs) is an established method used to study early development, physiology, disease and neurotoxicity (Riemens et al., 2018). However, there is a need for robust protocols that systemically characterize cells at intermediate differentiation timepoints. These types of in vitro studies should offer the cell-type resolution necessary to characterize developmental trajectories. Improving the understanding of a protocol's ability to recapitulate early brain development will aid future studies and increase applicability.

The role of epigenetic regulation on the establishment and maintenance of cellular identity during early neuronal differentiation processes is not well understood (Sun et al., 2021; Yao et al., 2016). Therefore, in-depth analyses describing epigenetic landscapes and the complex interplay with gene expression are required. Moreover, mapping derivative cells and their development- or region-specific transcriptional and epigenetic landscapes is fundamental for investigating disease mechanisms and for therapeutic interventions.

In this study, we used a multi-omics approach to construct a molecular timeline of early human neuronal differentiation. We used a novel 2D neuronal differentiation protocol using dual SMAD/WNT signalling inhibitors LDN193189, SB431542 and XAV939 (LSX) for neural induction of human embryonic stem cells (hESCs) (Cakir et al., 2019; Chavali et al., 2020; Major et al., 2016; Ohashi et al., 2018; Tchieu et al., 2017). The neuronal progenitors were allowed to self-pattern and mature towards a heterogenous population of immature and mature neurons bearing telencephalic signatures. We performed RNA-seq, global DNA methylation, single-cell RNA-seq and ATAC-seq data integration across timepoints (4D analysis), to correlate the expression of transcription factors with time- and population-specific chromatin states in hESCs, and during differentiation. This integration of comprehensive multi-omics data enabled the characterization of both the transcriptional and epigenetic landscapes in this model of early fate commitment. We provide access to single-cell data in user-friendly, interactive web applications that enable visualization of gene cluster regulation during the neuronal differentiation protocol. 


\section{Results \\ Initial validation of the neuronal differentiation protocol}

The coating conditions and cell numbers were optimized to permit high cell contact, proliferation, and viability. Thus, based on confluency, morphology and viability, we analyzed the hESCs (Day 0) and derivative cell populations at three timepoints. We defined the end of the neural induction phase (Stage I) at Day 7 , the end of the selfpatterning phase (Stage II) at Day 13 and at the end of the maturation phase (Stage III) at Day 20 (Fig. 1A). For the neural induction of undifferentiated HS360 hESCs (Main et al., 2020; Ström et al., 2010), we used LDN193189, SB431542 and XAV939 (LSX). This LSX cocktail antagonizes the BMP, TGF $\beta$ and WNT signalling pathways to drive cells to anterior neuroectoderm (Cakir et al., 2019; Major et al., 2016; Ohashi et al., 2018; Tchieu et al., 2017). By the end of Stage I, neural induction morphogenetic events shape cells into thickened neural rosettes, whereas at Stage II, cells self-pattern before the Stage III FGF2/EGF maturation phase (Fig. 1B). In the absence of inhibitors at the self-patterning stage II, the cells retain their anterior forebrain identity and proceed to maturation, as shown by the ddPCR results (Fig. 1C).

The expression of the pluripotency markers POU5F1 and NANOG decreased significantly after neural induction $(p<0.00001)$. Expression of the early neural markers SOX2 and NES increased and stabilized at Day 7, whereas PAX6 expression peaked at Day 7 before decreasing significantly at Days 13 and $20(p<0.0001)$. The expression of the transcription factor (TF) OTX2, which regulates neurogenesis and antagonizes ground state pluripotency, the late onset pan-neuronal marker TUBB3, and also MAP2 and FOXG1 increased as cells differentiated. Immunofluorescence imaging showed protein expression and localization of OCT4, OTX2, SOX2, PAX6, NES and TUBB3 (Fig. S1). 

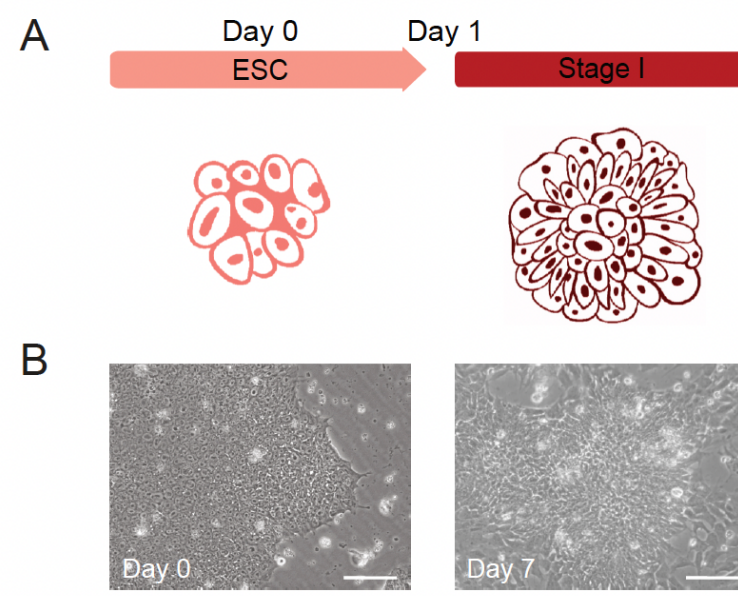

Day 7

Day 13

Day 20

\section{C}
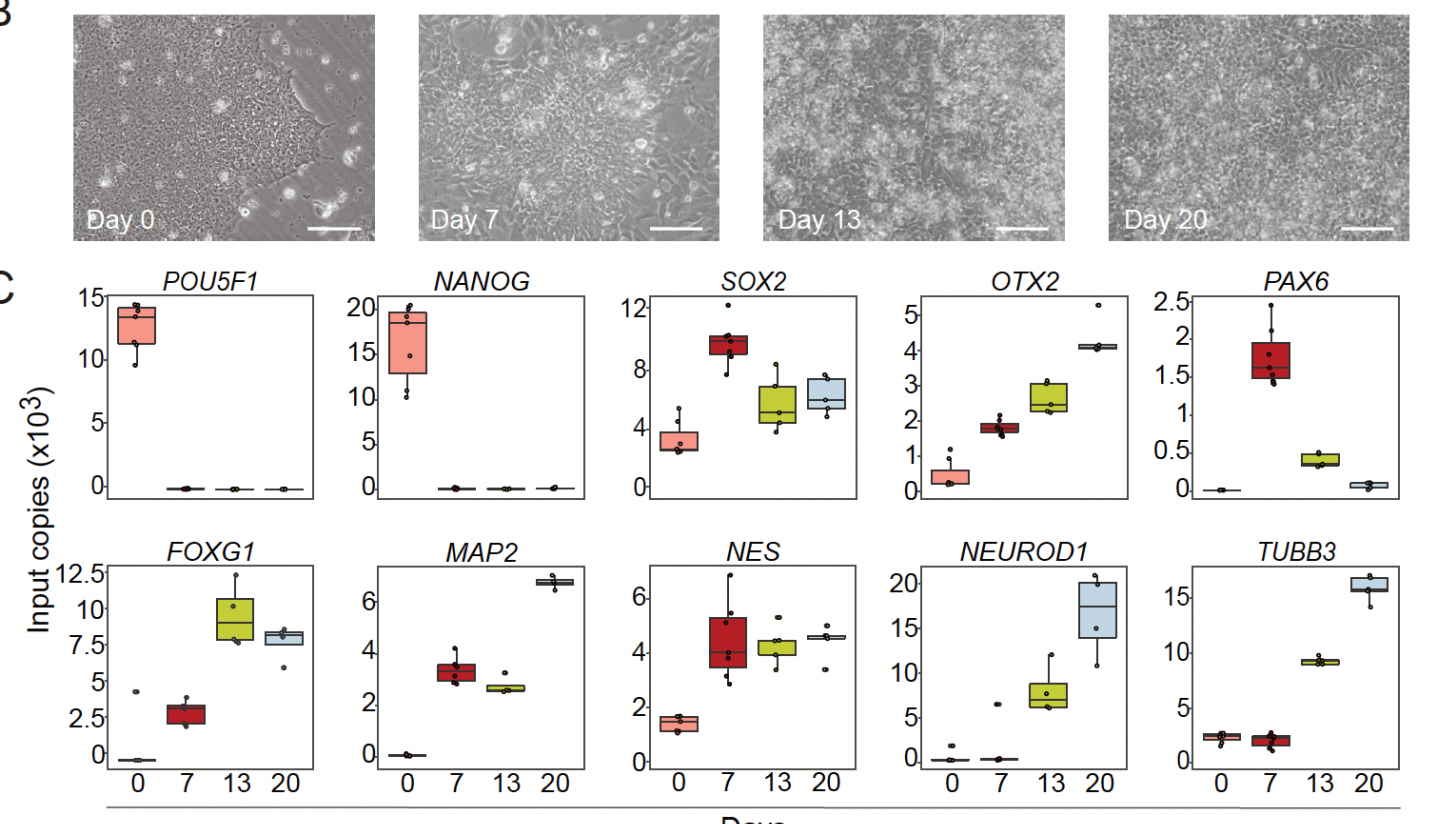

NANOG
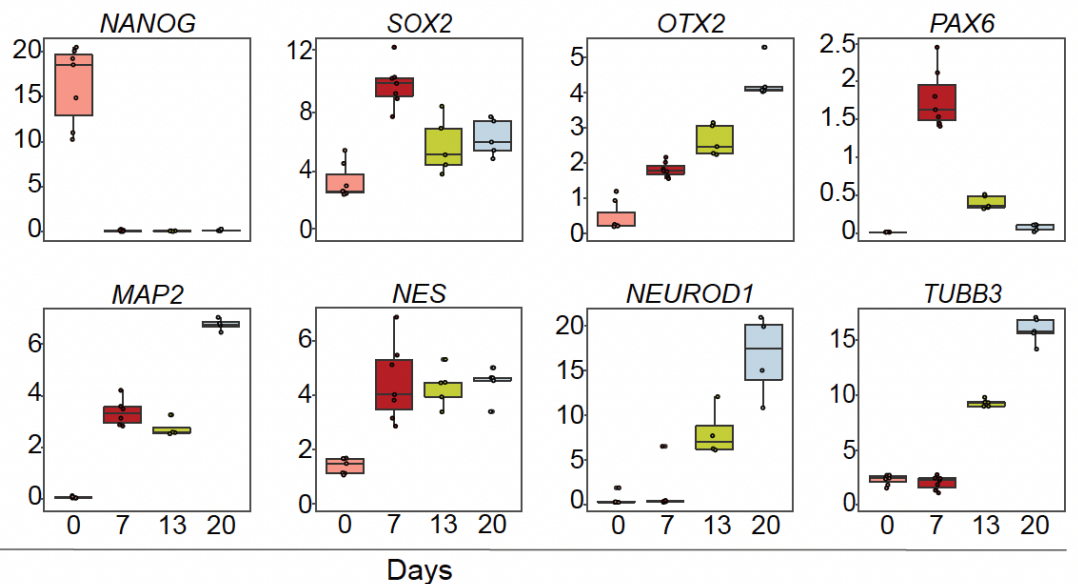

Figure 1. 2D protocol with neural induction followed by self-patterning and maturation. A) Schematic illustration of the 20 Day timeline of the neuronal differentiation protocol from hESCs. B) Representative 20x brightfield phase contrast images of hESCs at Days $0,7,13$ and 20 (scale bar $100 \mu \mathrm{m}$ ). C) ddPCR results from 4-6 replicates of mRNA expression of selected marker genes from Days 0, 7, 13 and 20.

\section{Identification of heterogenous populations of progenitors, mature and immature} neurons with telencephalic signatures

To characterize the gene expression signatures, composition, differentiation pathway trajectories and the maturation level of the cell types derived, we performed single-cell RNA-seq (scRNA-seq) analyses at Days 0, 7, 13 and 20 (Figs. 2, S2, S3 and Table S1). The scRNA-seq data can be visualized in the open access webtool "hESC Neuronal Differentiation scRNA-seq" (hESCNeuroDiffscRNA) where expression of genes can be explored per cell, cluster and timepoint (Star methods). A total of 9,337 cells were projected in UMAPs, 1,900 Day 0 cells, 2,368 Day 7cells, 2,045 Day 13 cells and 3,024 Day 20 cells). (Fig. 2A and hESCNeuroDiffscRNA, cell information tab, orig.ident). 


\section{Inferring quantitative analysis of cell cycle phase}

A hallmark of neuronal development involves major alterations in G1- and S-phase duration. G1-phase lengthening is associated with the transition to a more differentiated cell type, while S-phase duration is linked to progenitor cell expansion (Arai et al., 2011). The cell cycle-specific gene trajectories showed a transition from $15.4 \%$ to $54.1 \%$ cells in G1 phase from Day 0 to 20 (Fig. 2B, S2D, Table S2 and hESCNeuroDiffscRNA). This is consistent with previous studies showing that the maintenance of pluripotency, proliferation and differentiation of rapidly proliferating PSCs, neural stem cells and progenitor cells are regulated by the cell cycle (Becker et al., 2006; Boward et al., 2016; Liu et al., 2019; Soufi and Dalton, 2016). The cell cycle regulator CDK1 was expressed in $60 \%$ of the cells at Day $0,45 \%$ at Day $7,53 \%$ at Day 13 , and reduced to $33 \%$ at Day 20 (Fig. S2E, \% from the hESCNeuroDiffscRNA). CytoTRACE results confirmed that differentiation is consistent with the cell cycle phase inferred trajectory. As indicated by the higher CytoTRACE scores, cell potency gradually decreased from Day 0 to 20 (Fig. 2C), confirming the cell cycle phase prediction.

\section{Development and differentiation markers used for cluster resolution and annotation}

The four timepoints were resolved into 15 clusters (R1-R15, Fig. 2D). Corresponding cell numbers per cluster and cells per timepoint per cluster are shown (Table S3). The top ten most highly expressed genes for each cluster are plotted in a heatmap (Fig. 3), including many developmentally regulated TFs. Among these genes, POU5F1, TDGF1, GAL, LRRC75A, RAX, LIX1, TYMS, HES1, HES5, HES6, FGF17, DLX5, $D L X 6, G A P 43, S T M N 2$ and GNRH1 were used for R1-R13 cluster annotation. For R14, consisting of 27 Day 0 cells, we used KPNA2, a gene associated with the localization of OCT4 (Li et al., 2008). For R15, a pool of 90 cells from Days 7, 13 and 20, we used FABP7, which is expressed in NSCs during development (Kurtz et al., 1994).

\section{Characterizing the unsynchronized hESC population}

We identified three distinct Day 0 clusters (R1-3) where all cells expressed POU5F1, verifying their pluripotency. TFs essential in establishing and maintaining pluripotency (i.e., GAL, TDGF1, ID1, FOXH1 and SOX2) were highly expressed in clusters R1-3 (observe in hESCNeuroDiffscRNA). R3 cells expressed the highest levels of NANOG and $L R R C 75 A$. As others have reported (Chen et al., 2021), PHC1 was highly expressed in hESC clusters R1-R3, and its expression was greatly reduced in differentiating cells. Downregulation of PHC1 was compensated by increased PHC2 expression, indicating a role for PHC2 in human neuronal differentiation (Fig. 2E). Focusing on the FOX family of TFS, FOXD3, which is required for pluripotency (Krishnakumar et al., 2016), and the recently reported pluripotency marker FOXD3AS1 (Haswell et al., 2021), were expressed in R1-R3 (Fig. S3A). Furthermore, a clear switch was observed from FOXH1 and FOXD3-AS1 expression in R1-R3, to the 
expression of the master regulator of brain development FOXG1 (Beyer et al., 2013; Chiu et al., 2014) in all other clusters (Fig. 2E and S3A).

A
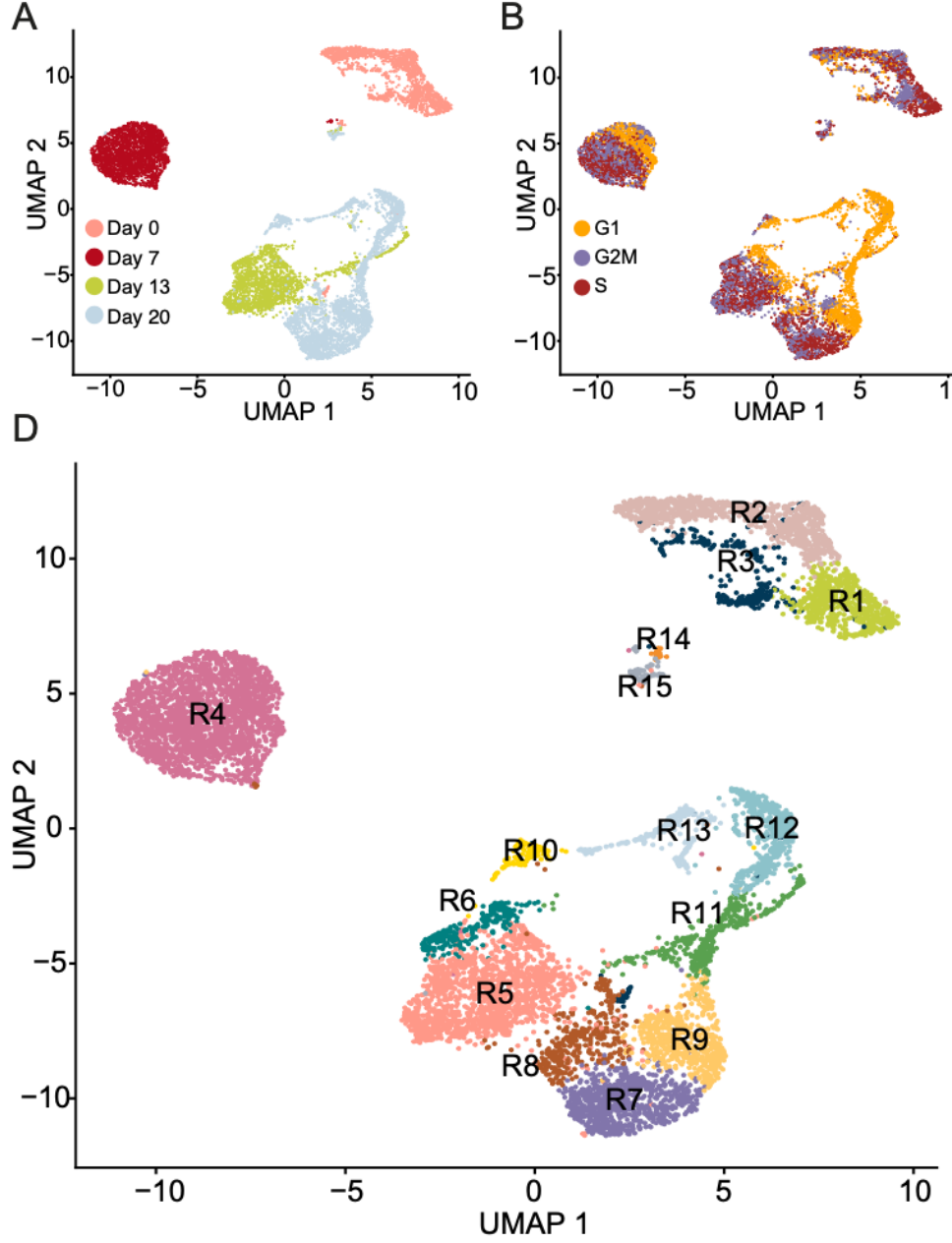

B

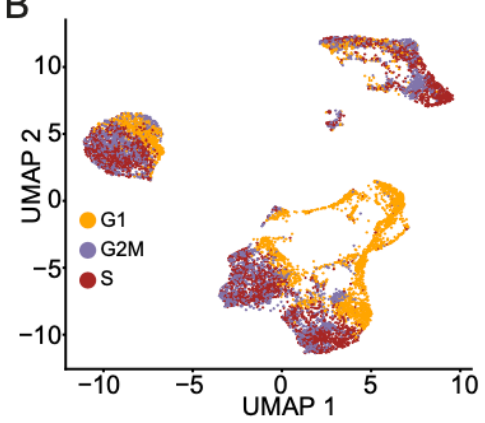

R1 POU5F1/TDGF1/GAL/TAGLN/

- R2 POUSF1/TDGF1/GALID1

- R3 POU5F1/TDGF1/GALLLRRC75A

- R4 PAX6/RAX/SOX2/LIX1

- R5 REST/HES1/FABP7/BMP4

- R6 REST/HES1/BMP4/DLX5

- R7 REST/HES5/TYMS/KIF15/CDK1

- R8 REST/HES1/FGF17

R9 REST/HES5/ASCL1/CDK1-

R10 REST/DLX5/DLX6/BMP4

- R11 CDKN1C/NEUROG1/HES6

- R12 NTRK1/STMN2/ISL1/L1CAMI

AP43/ELAVL4

R13 GNRH1/DLX6-AS1/GAD2/ARX

R14 KPNA2

R15 FABP7

E
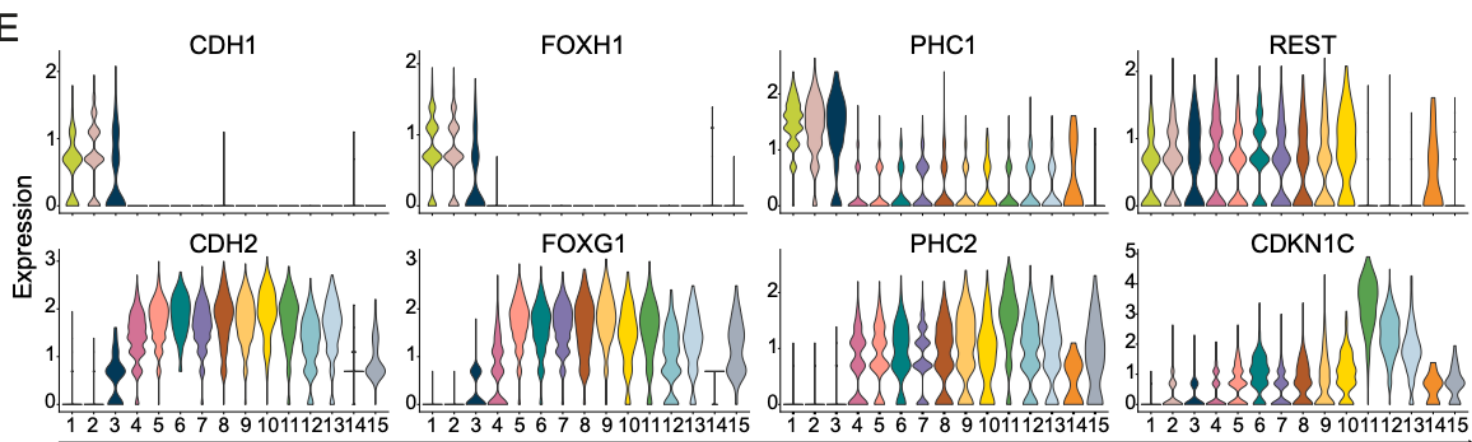

PHC2

CDKN1C

23456789101112131415 i

Figure 2. Identification of cell populations during neuronal differentiation of hESCs. A) UMAP representing single-cell RNA-seq clusters per time-point. B) UMAP of cell cycle analysis showing all cells analyzed and coloured by assigned cell cycle phase. C) The inferred neuronal differentiation trajectory using CytoTRACE, where less differentiated cells are shown in red and more differentiated cells are shown in blue. D) The UMAP representing cell clusters R1 to R15 with corresponding gene annotations mapped at resolution 0.55 . The clusters are indicated by different colours and gene annotations per cluster are given after each colour corresponding bullet 
below the UMAP. E) Violin plots representing gene expression levels and distribution in clusters R1 to R15 for selected genes.

\section{LSX forebrain induction cues evident at the end of Stage I}

Under LSX induction, hESCs undergo morphogenetic events and form neural rosettes. These Day 7 cells mapped to a single cluster (R4, Fig. 2D), enriched in the rostral markers SIX3 and LIX1 (Figs. 3, S2E and S3D). In contrast, the expression of the preplacodal genes EYA1 and SIX1 (Ikeda et al., 2007; Schlosser, 2014) was low, including SIX3-AS1. Furthermore, expression of caudal markers PAX5 and GBX2 (Kirkeby et al., 2012; Maroof et al., 2013) was low throughout differentiation. This confirms the efficacy of LSX forebrain induction, enabling cell-fate commitment persistence.

Upon neural induction, the key neural TF PAX6 is upregulated and interacts with SOX2 (Zhang et al., 2019). R4 cells were SOX2 positive showing high PAX6 and RAX expression (Fig. S2E), and express neuronal rosette markers, such as DACH1, POU3F2, NR2F1 and NR2F2 (Fedorova et al., 2019). A distinct switch from CDH1 (Epithelial Cadherin) to $\mathrm{CDH} 2$ (Neural Cadherin) expression was observed (Fig. 2E), and other developing forebrain specification and differentiation stage markers (such as OTX2, HESX1, FOXG1, LIN28A and FABP7) were detected.

\section{Self-patterning does not affect fate commitment}

At Day 13, which marks the end of the self-patterning stage, $75 \%$ of the cells mapped to cluster R5 and most of them expressed REST (Figs. 2E and S2E). SIX3, DLX5 and BMP4 (Figs. S2E and S3D) were expressed in R6 cells that are enriched in FGF8 and HES1. Moreover, R6 was enriched in TAGLN, which was also expressed in $44 \%$ of R5 cells but absent in the R5 cells co-expressing NKX2.1, DCT and SOX6. CNTN1, an active ligand of Notch (Hu et al., 2003) and potent inducer of neuronal migration (Lee et al., 2014), was expressed in R6. CNTN1 was exclusively expressed in cells negative for NTN1, DLL1, FABP7 and POU3F2. Comparing to results of 8 week human embryonic tissue (Kirkeby et al., 2012), no midbrain and hindbrain markers were detected at Day 13, confirming that the self-patterning phase does not affect fate commitment.

\section{Characterization of the Day 20 heterogenous population}

Day 20 cells retained their identity and clustered in R7-13 (Figs. S2E and 3). Some cells expressed high levels of CDK1 (Fig. S2E), whereas other cells were still regulated by REST and expressed $D L X 5$ and CDKN1C. CDKN1C, which forms complexes with histone deacetylases to repress neuronal genes in non-neuronal cells (Laukoter et al., 2020 ) is inversely correlated with REST expression and enhanced in R11-13 (Figs. $2 \mathrm{E}$ and 3 ). Interestingly, $A R X$, a regulator of cortical progenitor expansion by repression of CDKN1C (Colasante et al., 2015) was only expressed in R13 cells (Fig. S2E). Neuronal differentiation correlated with CDK6 upregulation and G1 shortening. CDK6 is directly regulated by GLI3 and expression of GL/3 (Hasenpusch-Theil et al., 2018)(detectable at R4) dropped significantly in R12-13. REST is known to be 
downregulated during neurogenesis and in differentiating neurons and the pattern was recapitulated in this study (Fig. 2E).

\section{Neuronal maturation signatures}

Day 20 cells were highly enriched for MAP2, and clusters R11-13 were enriched for $D C X$, which is a marker of migratory neurons. Genes expressed in proliferating neuroblasts associated with cortical migration control and developing rostral brain structural patterning, such as EMX2 (Pang et al., 2008; Spalice et al., 2009; Verrotti et al., 2010), decreased in clusters R11-R12 and were undetectable in R13 (Fig. S2E). FGF8, an anterior-posterior patterning molecule, acting mainly via EMX2 repression (Hao et al., 2019), was expressed in R4 and R6 cells and in a few Day 20 cells, mainly in R8 and R10 clusters. Furthermore, FGF17 (Figs. 3 and S2E) and FGF18 were mostly expressed at Day 20 R8 cluster. HES6-enriched cluster R11 (Fig. S2E) was composed of Day 13 and 20 cells, and most of the R11 NEUROG1-negative cells were Day 13 cells. Neural stem and progenitor marker ZEB1, which was downregulated upon neuronal differentiation to permit proper migration of immature neurons (Wang et al., 2019a), was expressed in almost all cells (Figs. 3 and S2E). In addition, FOXG1enriched R13 cells also express high levels of $D L X 6-A S$ and $D L X 5$ (Figs. 3, S2E and S3B).

Of note, the expression of GNRH1 (Gonadotropin Releasing Hormone 1) was expressed in $30 \%$ of the R12-13 cells (9\% of Day 20 cells) (Figs. 3 and S2E). Of these cells, some expressed GABAergic or glutaminergic processing enzymes. As the mechanisms that contribute to the development of extrahypothalamic $\mathrm{GnRH}$ neurons are not fully described, such data are vital for studies of development, puberty and reproduction. 


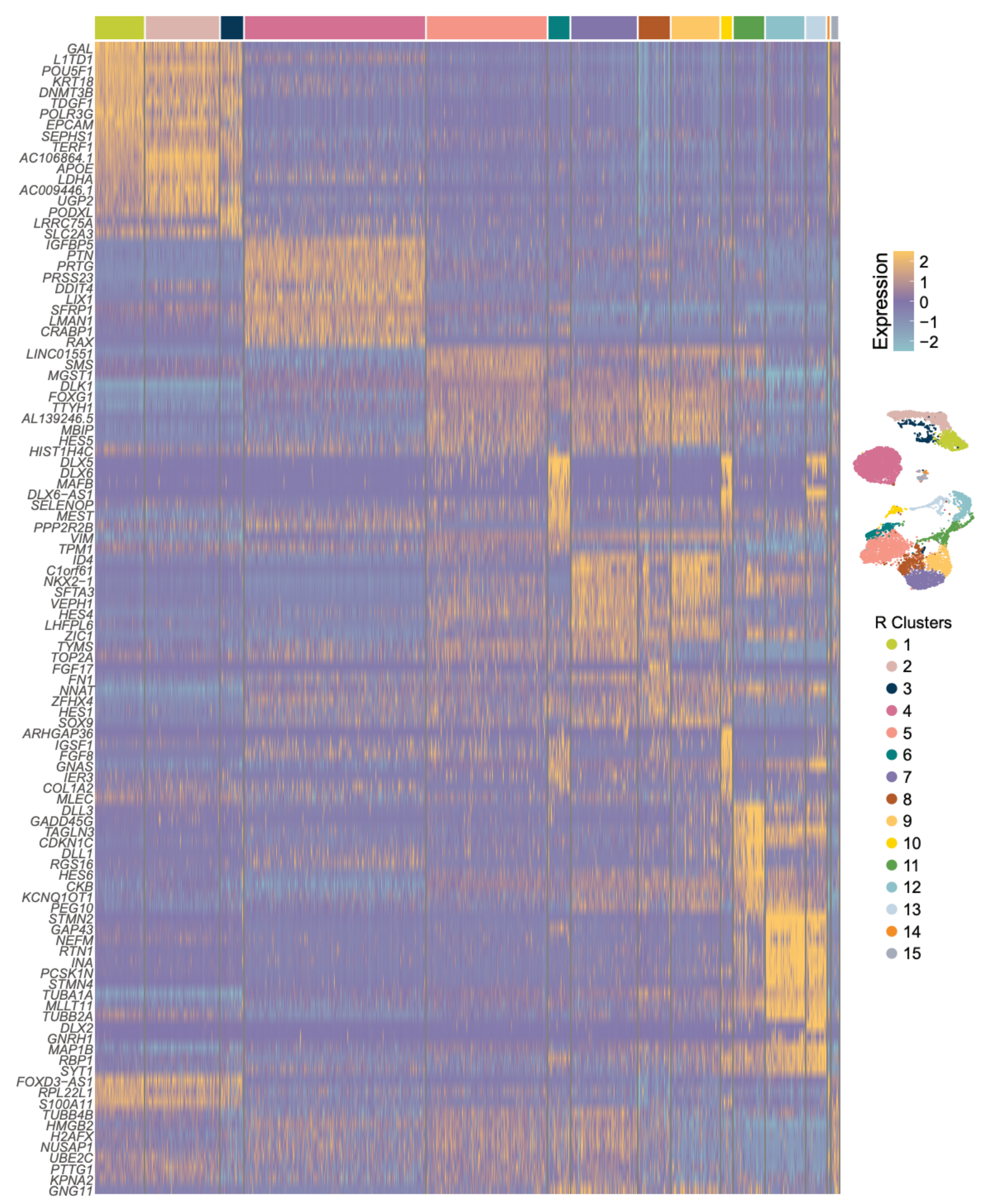

Figure 3. Cluster analysis of differential gene expression. Heatmap of the top ten most highly expressed genes in all clusters. Rows represents single genes and column represents single cells. Cell clusters are ordered sequentially and coloured according to the UMAP annotation shown to the right (yellow represents high expression and blue low expression). 


\section{Global expression profiles reveal neuronal differentiation and maturation signatures}

To increase gene expression sensitivity, we performed bulk gene expression analysis with higher sequencing depth (Fig. 4 and S4). Overall, we found 11,313 differentially expressed genes (DEGs) comparing cells from Day 0 to 20 (Table S4). More genes were differentially expressed during neural induction (Day 0 to 7 ), compared to the later stages, with self-patterning (Day 7 to 13) and maturation (Day 13 to 20) stages (Table S4). The most extensive transcriptional changes occurred between Day 0 and Stage I, with loss of pluripotency and gain of neuralization markers (Figs. 4A, S4A and S4F). We confirmed that bulk RNA-seq analysis for selected marker genes correlates well with ddPCR (Fig. 4B and S4G).

The analysis shows the specific gene expression patterns as cells lose pluripotency and move towards neuronal maturation (Fig. 4). These may be steep decreases after neuronal induction, as seen for $L I N 28 A$ and $C D H 1$. For other genes expression peaks at Day 7 or Day 13, such as $R A X$ and FOXG1, respectively. Expression increases gradually for genes such as OTX2 and SOX6. Moreover, the expression of neuroectodermal patterning Wnt/ $\beta$-Catenin negative regulator AMER2 (Pfister et al., 2012), and neuronal differentiation marker STMN2 (Wang et al., 2019b) increase at Day 13 and increase further at Day 20 (Fig. 4A). On Day 20 we also find genes correlated to specific neuronal types, such as GRIA1, SLC17A6, GNRH1 and GAP43 (Fig. 4A and S4F).

We next performed gene ontology (GO) analyses to identify shared biological processes (BP) among the DEGs during differentiation (Fig. 4D and Table S5). These analyses revealed enrichment of upregulated BPs related to pattern specification, neuronal maturation and migration from Day 0 to 20 (Fig. 4D). Stage-specific GO analyses revealed enrichment of BPs involved in neurogenesis and neuron development differentiation at stage I (Day 0 to 7), and BPs involving synaptic organization and signalling, and neurotransmitter regulation and secretion at the end of the maturation stage (Day 13 to 20; Table S5). The RNA-seq analysis is in line with single-cell analysis showing downregulation of pluripotency genes and upregulation of brain development genes. 
A
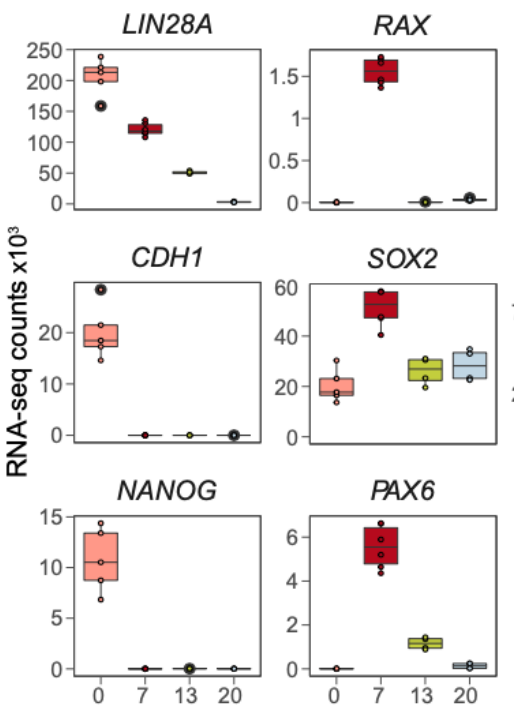

SOX2

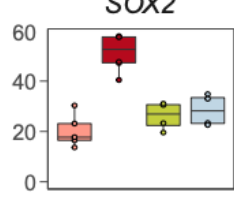

PAX6
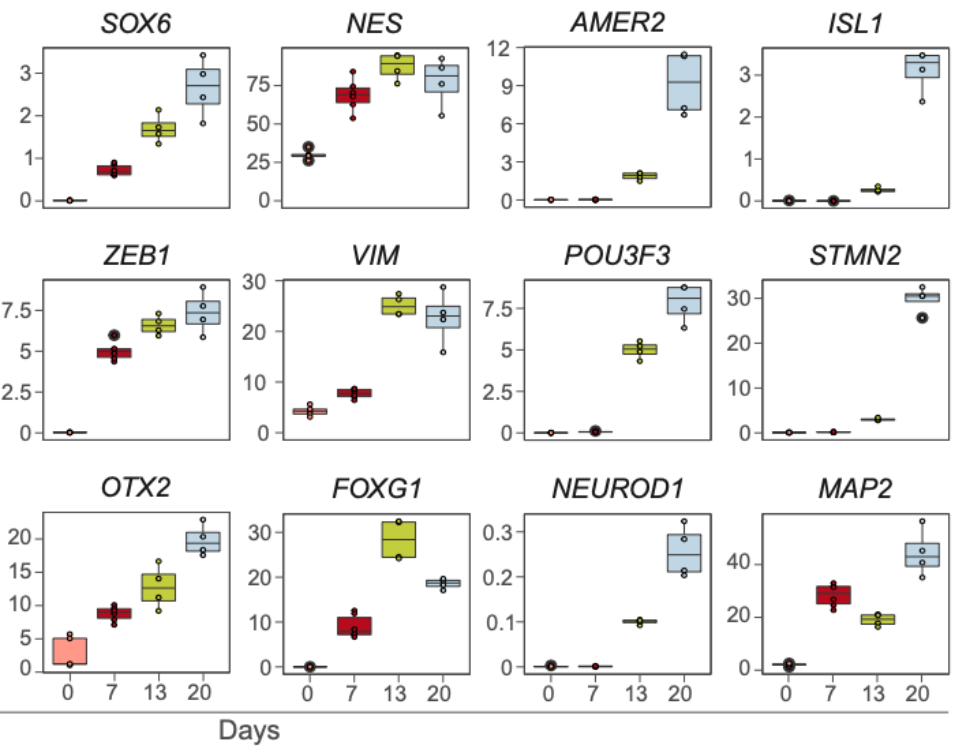

NEUROD1
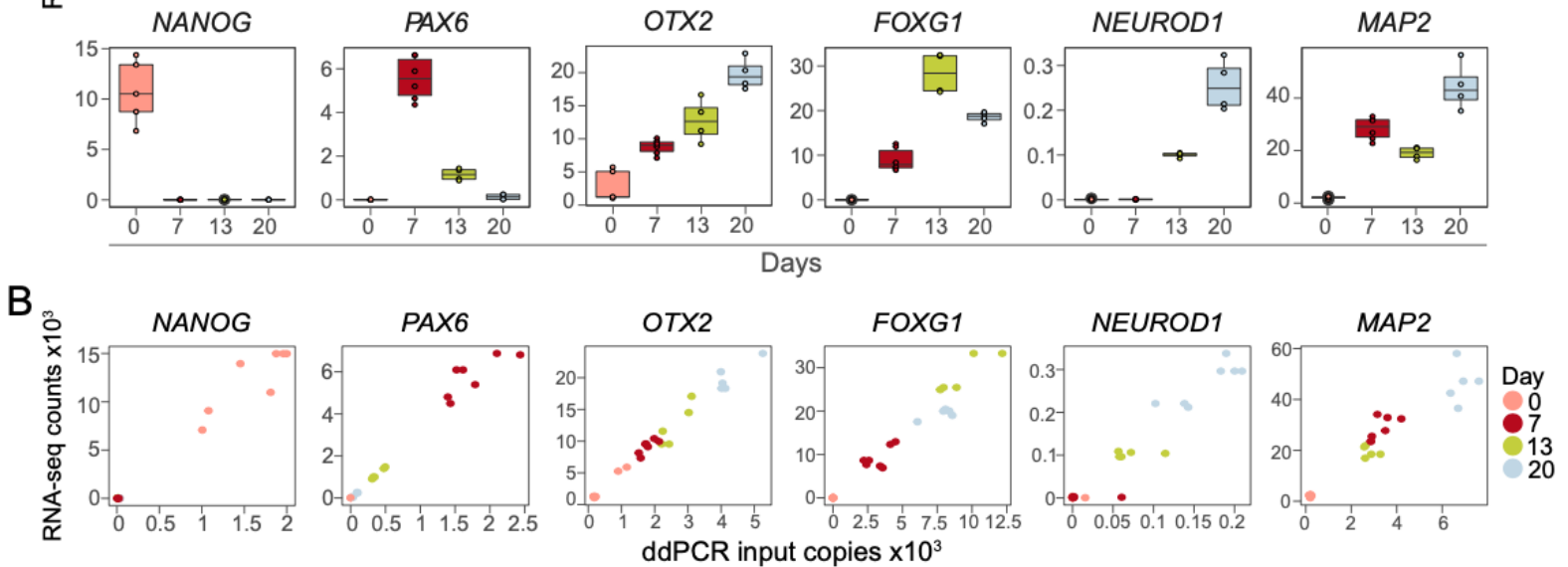

C

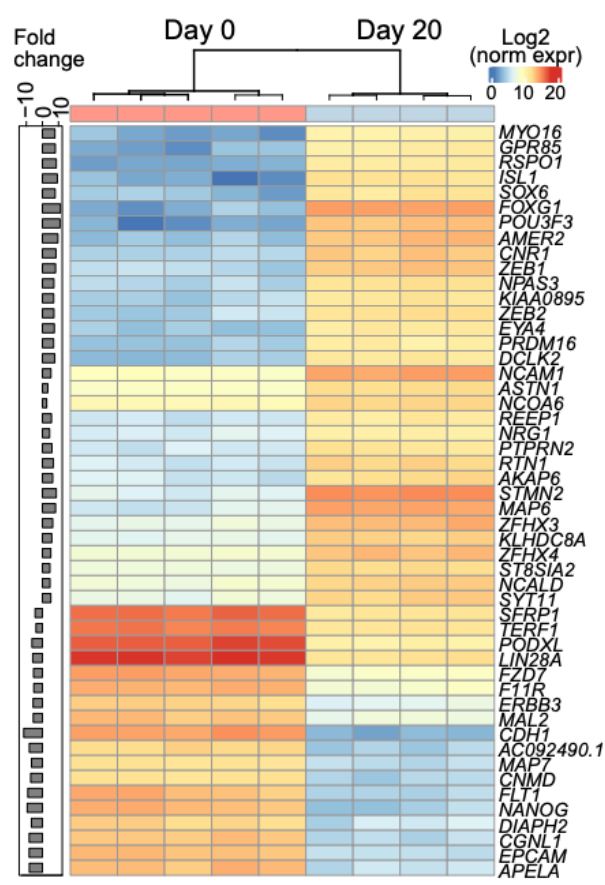

D

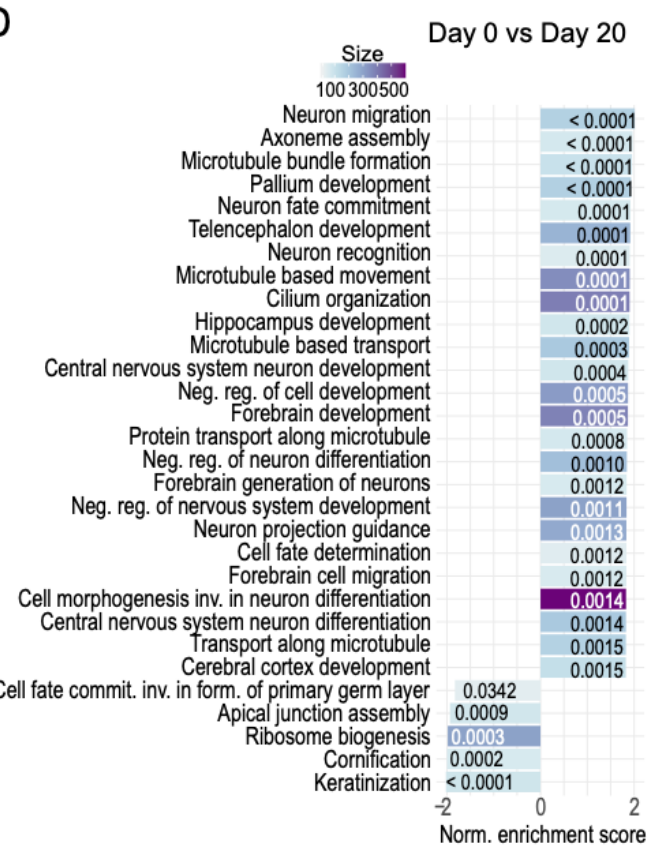

Figure 4. Correlation of RNA-seq to ddPCR and GO analyses results. A) Normalized gene expression counts for selected genes showing transcriptome expression patterns from loss of pluripotency towards neuronal maturation. B) Scatter plots of RNA-seq and ddPCR for marker genes NANOG, PAX6, OTX2, FOXG1, NEUROD1 and MAP2 at Days 0, 7, 13 and 20. C) Heatmap of top 50 differentially expressed genes between Days 0 and 20 replicates. Fold change is shown to the left. D) GSEA analysis of differentially expressed genes from Days 0 and 20. 


\section{DNA methylation correlates with neuronal transcriptional programs during differentiation}

DNAm in human cells is mainly restricted to CpG sites and essential for normal development (Smith and Meissner, 2013). As hESCs transition to differentiated neurons, dynamic DNAm changes regulate gene expression and the establishment of cell-type specificity (Stricker and Götz, 2018). To assess DNAm in the present protocol, we identified $\mathrm{CpGs}$ which are differentially methylated (DMCs) between Day 0, 7, 13 and 20 (Figs. 5 and S5). As expected, comparing Day 0 and 20 reveals massive DNAm changes ( $n=210,049$ DMCs, Table S4). Although we observe major changes in DNAm during the differentiation protocol (Table S4), the bulk DNAm levels and the distribution of unmethylated and methylated $\mathrm{CpGs}$ remains the same across all four timepoints (Figs. 5A and S5B-C).

Deconvoluting these changes temporally, the highest number of DMCs was observed between Day 0 and $7(n=161,600)$, with fewer changes in the self-patterning phase (Days 7-13, $n=39,545$ ) and during cell maturation (Days 13-20, $n=47,676$ ) (Table S4). Next, we used GOMETH analysis (Maksimovic et al., 2021) to explore shared biological functions among the DMCs. In line with the gene expression results, from Day 0 to 20 we observed enrichment of BPs involved in neural induction, neurogenesis, and brain development (Fig. 5C). This suggests that DNAm is modulating the neuronal transcriptional programs during the course of differentiation (Fig. 5A). Similarly, these analyses identified DMCs between Day 0 to 7 and Day 0 to 13, with BPs involved in cell adhesion and neuron projection morphology, which fits well with the stage cell transitions (Fig. S5D and E). One of the most significant GO terms is "neuron migration", evidenced by expression of genes such as $D C X$ and its partner PAFAH1B1 (Nadarajah and Parnavelas, 2002) (Fig. 5C), both highly expressed in R11-13 (Fig. 2 and hESCNeuroDiffscRNA).

To explore the correlation between DNAm and gene expression, we combined the DNAm and RNA-seq data sets based on CpG probe location and gene locus (Figs. 5D, S57-G, and Table S4). Of the Stage I gene annotated DMCs, 72\% overlap with differentially expressed genes, inferring functional impact on gene expression. For genes with DMCs we generally observed a decrease upon transcriptional activation or an increase for genes becoming repressed during the course of differentiation. The expression levels of the majority of the differentially expressed genes between Day 0 and 20 are predicted to be associated with DNAm changes $(8,011$ of the 11,313 DEGs). The expression of markers of late trophectoderm (e.g., KRT18), pluripotency maintenance (POU5F1), suppression of pluripotency (NR6A1) (Wang et al., 2016), metabolic reprogramming (LDHA) (Zheng et al., 2016), or spatiotemporally regulated cortical TFs and cell cycle related genes (LHX2, CDKN1C) (Chou and Tole, 2019; Laukoter et al., 2020); and neuronal differentiation and maturation markers, (such as $D C X)$, may be regulated by one or more CpGs (Fig. 5C, S5F, S5G and Table S6). 
Of note, the average non-CpG DNAm levels, and the distribution of unmethylated and methylated $\mathrm{CpHs}$ vary across time points. $\mathrm{CpH}$ DNAm is associated with transcriptional repression in the mouse genome (Xie et al., 2012) and non-CpG DNAm levels are enhanced at Day 0 cells and decline during differentiation (Fig. 5B).

A

B
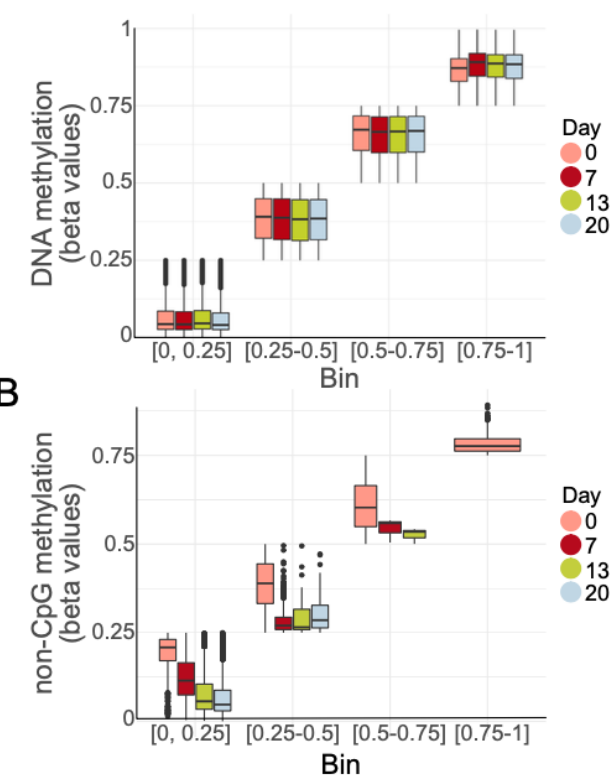

C

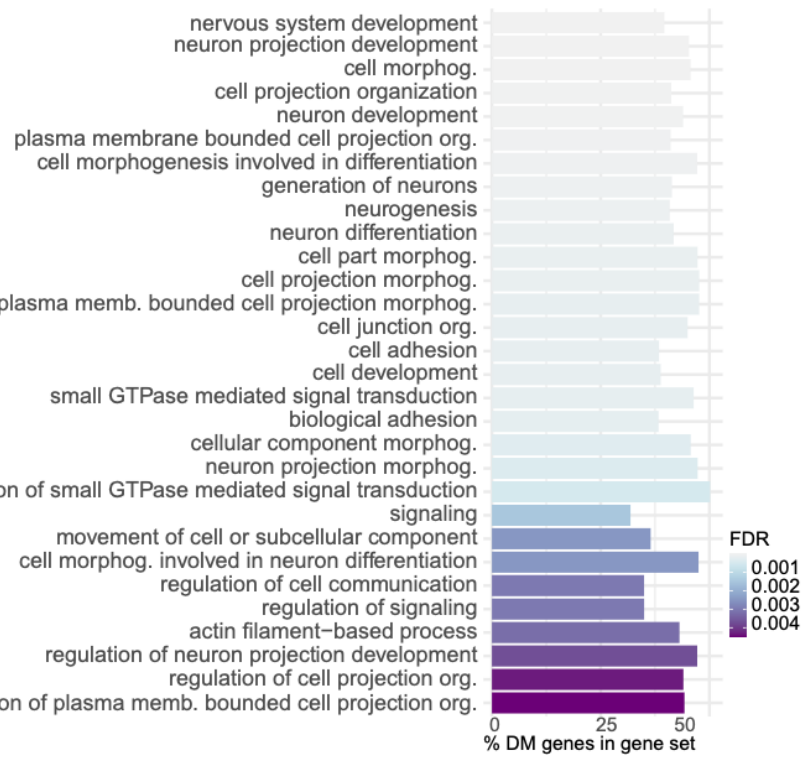

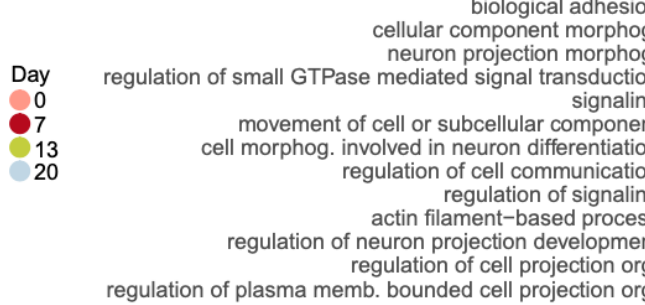

$\%$ DM genes in gene set

Day 0 vs 20
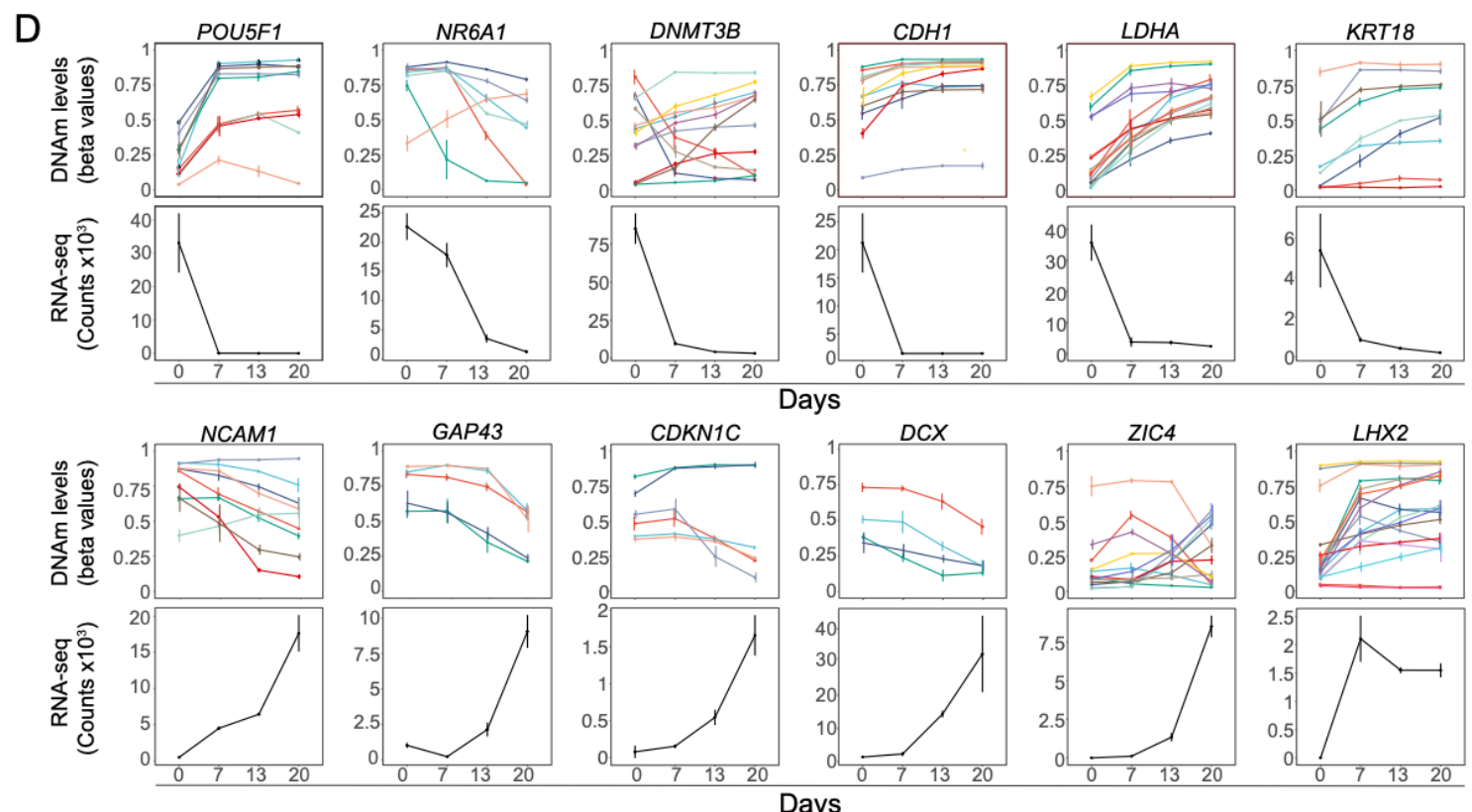

Figure 5. Specific DNAm changes during neuronal differentiation

A) Mean DNAm levels for each sample across all CpGs and non-CpGs (grouped in bins of 0.25 ) at Days $0,7,13$, and 20. B) Mean DNAm levels for each sample across all non-CpGs (grouped in bins of 0.25 ) from Days 0, 7, 13 and 20. C) GOMETH analysis of top 30 BPs based on top 10\% DMCs for Day 0 to 7. D) Significant CpGs of gene expression (derived from MORE) for Days 0, 7, 13 and 20. Top panels show DNAm mean +/- standard deviation whereas bottom panels show normalized RNAseq counts for selected genes. 


\section{Chromatin accessibility analysis identifies regulation signatures during differentiation}

To further assess the changes in the epigenetic landscape upon differentiation, we performed single-cell assay for transposase accessible chromatin sequencing (scATAC-seq). This analysis aimed at understanding chromatin-based gene regulation during neuronal differentiation from the loss of pluripotency at Day 0 to Day 20 (Fig. 6 and S6).

\section{Reanalyzing scRNA-seq datasets for integration with scATAC-seq data}

To integrate scATAC-seq and scRNA-seq, the scRNA-seq datasets for Day 0 and Day 20 were reanalyzed. 1910 Day 0, and 3033 Day 20 cells were projected in 14 clusters (Fig. 6A) and in accordance with the maturation trajectory seen in the corresponding CytoTRACE plot (Fig. 6B). The scRNA-seq clusters were numbered and annotated similarly to old clusters (Fig. 6A, S6A and B). Day 0 cells resolved into five clusters (R0-3 and R14) whereas Day 20 clusters were numbered as R7-15, cohering to the initial four-timepoint analysis.

\section{Chromatin accessibility changes globally during differentiation}

The analysis of 4,901 Day 0 nuclei and 2,847 Day 20 nuclei and the scATAC-seq data showed a good distribution of fragment sizes, fragment numbers, and TSS enrichment (Fig. S6C, D, E and F). The supervised pseudotime trajectory analysis, which predicts paths for gene regulatory changes in cells during differentiation, showed a similar profile to the gene expression CYTOTRACE analysis (Fig. 6B and C). We mapped four chromatin accessibility clusters at Day 0 (C1-4) and five at Day 20 (C5-9; Fig. 6D) and observed differential chromatin opening in these cell clusters for many loci, including POU5F1, REST, GAD2, and DCT (Figs. 2, S2, S3 and S6G). We next generated a gene score matrix heatmap incorporating regulatory elements, representing a score of chromatin opening of $200 \mathrm{~kb}$ gene regions. The heatmap shows a selection of marker genes based on their relevance to pluripotency and brain development and the previously described scRNA-seq cluster annotation (Fig. 6F). Higher gene scores for genes that are known for their role in the regulation of pluripotency, such as POU5F1, NANOG, ID1, and known enhancer specific binding factors in development, such as ZIC2 (Hong et al., 2011) were found in Day 0 clusters (Fig. 6F, 2 and S6B). SOX2 is regulated by several enhancers and interacts with multiple but distinct groups of transcription factors, including POU3 class partners (lida et al., 2020; Mistri et al., 2015; Tang et al., 2015; Zhu et al., 2014). Chromatin accessibility for SOX2, POU3F1/BRN1 and POU3F3/BRN3 increased with differentiation (Fig. 6F). Moreover, genes expressed at the differentiation endpoint clusters, such as ASCL1 and SOX21 which are implicated in neurogenesis (Fig. 2 and S3), NFIB which is crucial in neural progenitor cell renewal (Piper et al., 2014), and OTX2 which is associated with early neuronal development regulation, showed a more open chromatin structure in neuronal clusters C5-C9. The gene score of NEUROD1 is highest in the trajectory end-point cluster C5 (Fig. 6F). 


\section{Correlation of chromatin regulatory dynamics and gene expression}

To better understand the regulatory interactions with gene expression we performed integrated analysis of scATAC-seq with scRNA-seq using ArchR (Granja et al., 2021). Following constrained alignment of cell populations after integration of scATAC-seq and scRNA-seq, the integrated clusters were renamed to correspond to the previously annotated scRNA-seq clusters (Figs. 6E and S6B). Pluripotency clusters C1-C4 remapped to scRNA-seq Day 0 clusters R0 and R2, whereas clusters C7-C9 mapped to cluster R7, correlating chromatin openness and gene expression in single cells for markers such as REST, HES1, and CDK1 (Figs. 6B, D, E, S6B and S6I). Cluster C6 mapped to R9, which was marked by expression of REST, HES5 and ASCL1, and C5 remapped to one of the endpoint clusters, R12, having high NTRK1 expression (Fig. S6I). We assessed scATAC-seq peaks across TGDF1, CDH1, CDH2, STMN2, and $D C X$ loci across the integrated clusters and found cluster specific chromatin opening (Fig. 6I and S6J). Moreover, the peak-to-gene co-accessibility arcs show gene expression linked to chromatin opening during differentiation of putative distal regulatory elements at $T G D F 1$ and $C D H 1$.

To explore the integration of chromatin accessible regions and gene expression, we mapped 95,800 peak-to-gene links and observed a clear correlation of chromatin regulatory dynamics in the different integrated clusters (Fig. 6G). Moreover, chromatin accessibility was clearly enriched at transcriptional start sites (TSS) in every cluster (Fig. 6H). However, chromatin accessibility peak annotation analysis revealed varying enrichment across integrated clusters at promoters, intronic, exonic and distal regions (Fig. S6H). The number of peaks at promoters were very similar across the five clusters, with fewer peaks in cluster R9. Interestingly, more peaks were detected at intronic and distal regions in clusters R7 and R12 than in the other clusters. These results agree with previous studies showing that neuronal gene activation depends on multiple regulatory regions, many of which are located far from the gene locus itself.

We next aimed to explore the dynamics of lineage-defining factors at pluripotency and differentiation endpoint. Using ArchR, we identified specific TF motifs across differentiation (Fig. 6J). Motif footprinting for POU5F1 underlies a regulatory function in accessible chromatin in pluripotent clusters $\mathrm{R} 0$ and $\mathrm{R} 2$, whereas motifs for ASCL1 and OTX2 footprints were more enriched in differentiated clusters R7 and R12. We further mapped the enrichment of POU5F1, DLX6, ASCL1 and OTX2 motifs in open chromatin in individual cells (Figs. 6J and S6K). These examples illustrate how lineage-defining TFs dynamically regulate gene expression programs during neuronal differentiation. 

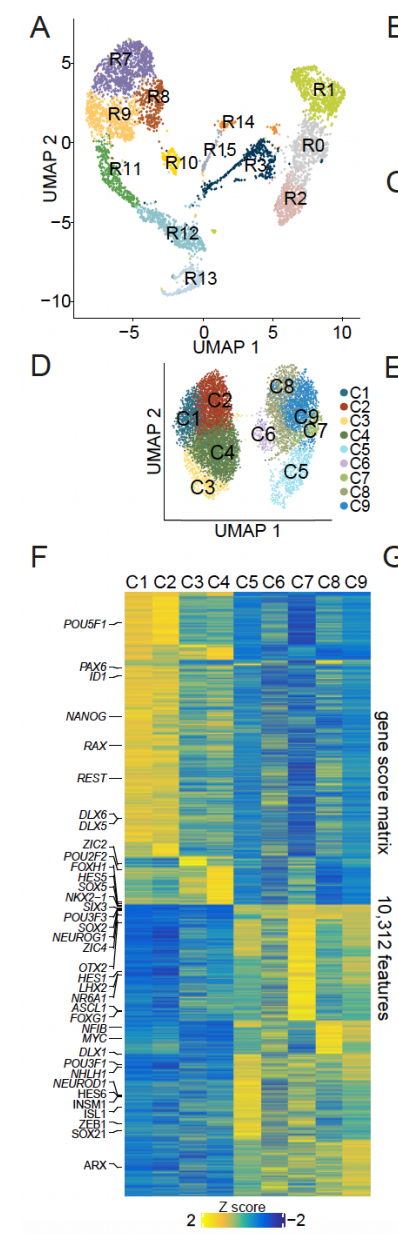

$\mathrm{H}$
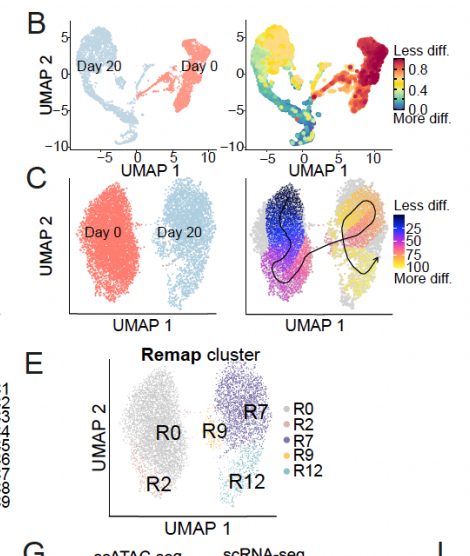

G

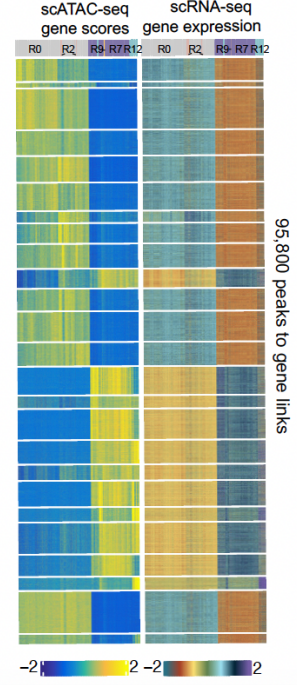

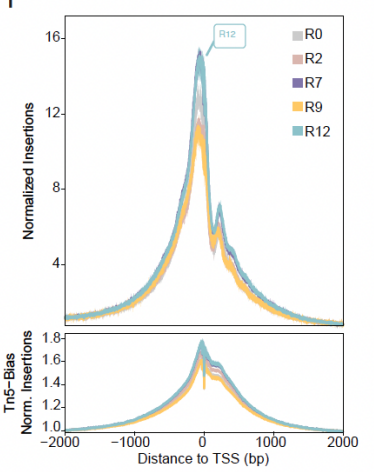

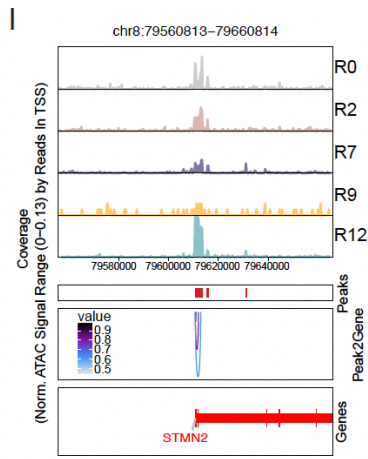

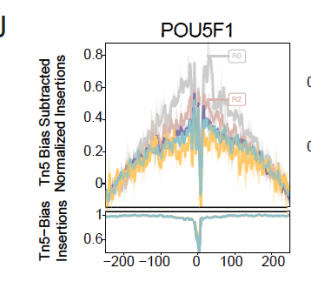

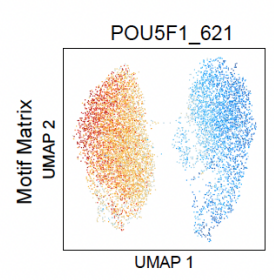

DevScores [-4 0

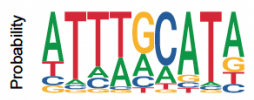
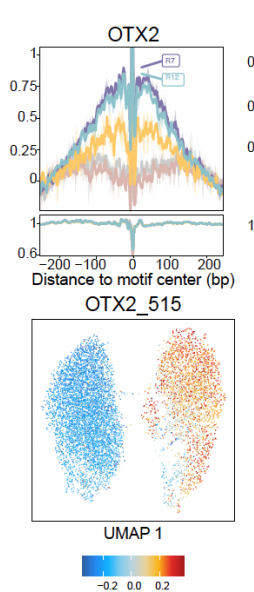

TTAATCT ACACTCTY

Figure 6. Integration of single-cell chromatin opening with scRNA-seq during neuronal differentiation. A) Multidimensional reduction UMAP plot of scRNA-seq corresponding to the timepoints used for scATAC-seq analysis. B) UMAP plot showing original identity of cells at scRNA-seq modality and corresponding differentiation trajectory. C) UMAP plot showing original identity of cells at scATAC-seq modality and corresponding supervised pseudo time trajectories. D) UMAP plot showing clusters at scATAC-seq modality. E) Remap UMAP plot of renamed clusters following constrained alignment of cell populations after integration of scATAC-seq and scRNA-seq. F) Top selected marker genes from scRNA-seq data shown on a heatmap plot computed on Gene Score Matrix. G) Peak to gene linkage heatmap for scRNA-seq clusters and corresponding gene scores for integrated scATAC-seq clusters. H) Chromatin openness of integrative cluster R0, R2, R7, R9 and R12 over all TSS. I) Tracks shown on peak browser for selected gene STMN2 on integrated cell clusters. Bottom panel shows co-accessibility interactions around TSS. J) Motif footprinting for selected transcription factors POU5F1, ASCL1 and OTX2 demonstrating preferential opening in different cell clusters. The middle panel shows the corresponding motif deviation scores of ArchR identified TFs POU5F1, ASCL1 and OTX2. The scores are calculated for each TF motif observed in an accessible region and in each cell for the deviation from expected average accessibility across all the cells. The representative sequence logos identified in accessible regions across the dataset are shown below. 


\section{Exploration of single-cell data using interactive webtools}

Large dataset analyses, such as single-cell sequence analysis, generally require bioinformatics expertise for interpretation. We have made our scRNA- and scATACseq data accessible to a broader audience by providing open access web-interfaces based on open-source tools, abiding by the Findability, Accessibility, Interoperability, and Reusability (FAIR) principles (Ouyang et al., 2021; Sharma et al., 2021). The users can explore scRNA-seq data in hESCNeuroDiffscRNA and plot high resolution figures of their genes of interest under seven different tabs (Fig S7A-H). This includes exploration of 1) Gene expression UMAPS as illustrated for POU5F1 and NTRK1;2) gene co-expression analysis, here shown for PHC1/PHC2 and NEUROG1/NTRK1; 3) different gene and cluster expression configurations, such as heatmaps, violin-, box-, proportion- and bubble plots. The platform also allows for correlation with other published gene expression datasets (Fig. S7H).

To illustrate the utility of the web interface, we focus on ZIC2 and ZIC4, and their expression and regulation during neuronal differentiation (Fig. 7). ZIC proteins are known for their role in proliferation and differentiation of neural progenitors, neurulation, neural tube formation, and neural plate closure (Al-Naama et al., 2020; Aruga and Millen, 2018). Global expression analysis shows that ZIC2 is present at Day 0 and peaks at Day 7, whereas expression of ZIC4, mostly undetectable at early timepoints, appears at Day 13 and peaks at Day 20 (Fig. 7A). DNAm levels at the CpGs in the ZIC2 locus were stable across differentiation. In contrast, DNAm of $13 \mathrm{CpGs}$ in the ZIC4 locus were positively or negatively correlated with gene expression across differentiation (Fig. 7B). Differential expression of ZIC2 and ZIC4 across the individual cells at Day 0, 7, 13 and 20 in UMAPs (Fig. 7C) can be compared and correlated with selected TFs, shown here to be important for regulation in the neuronal differentiation protocol (Fig. 7C).

The sCATAC-seq data can be explored in "hESC Neuro Differentiation scATAC seq" (hESCNeuroDiffscATAC) (Fig. S7I-N). Users can visualize chromatin accessibility, motif enrichment or integration of scATAC-seq with SCRNA-seq in UMAPs. Furthermore, this webtool enables investigation of the gene score and motif matrix, also showing the representative sequence logo calculated from open regions. (Examples are given for the L1TD1, HES5 and ZEB1 genes at Fig. S7J). Chromatin opening can be explored across the genome for different clusters, as shown for SOX2 and POU5F1 or in different heatmaps (Fig. S7K-N). Heatmap views could either be pseudotime trajectories or peak-to-gene linkage, which define linked chromatin opening peaks with promoters of expressed genes (Granja et al., 2021) and may deduce enhancer promoter interactions (Baek and Lee, 2020). In the case analysis of ZIC2 and ZIC4, gene score and gene integration analysis showed the genes were active in different cells (Fig. 7D) and the ZIC2 and ZIC4 footprints and representative sequence logos were identified. This 4D example analysis highlights the epigenetic regulation and gene expression of these genes in neuronal differentiation. 

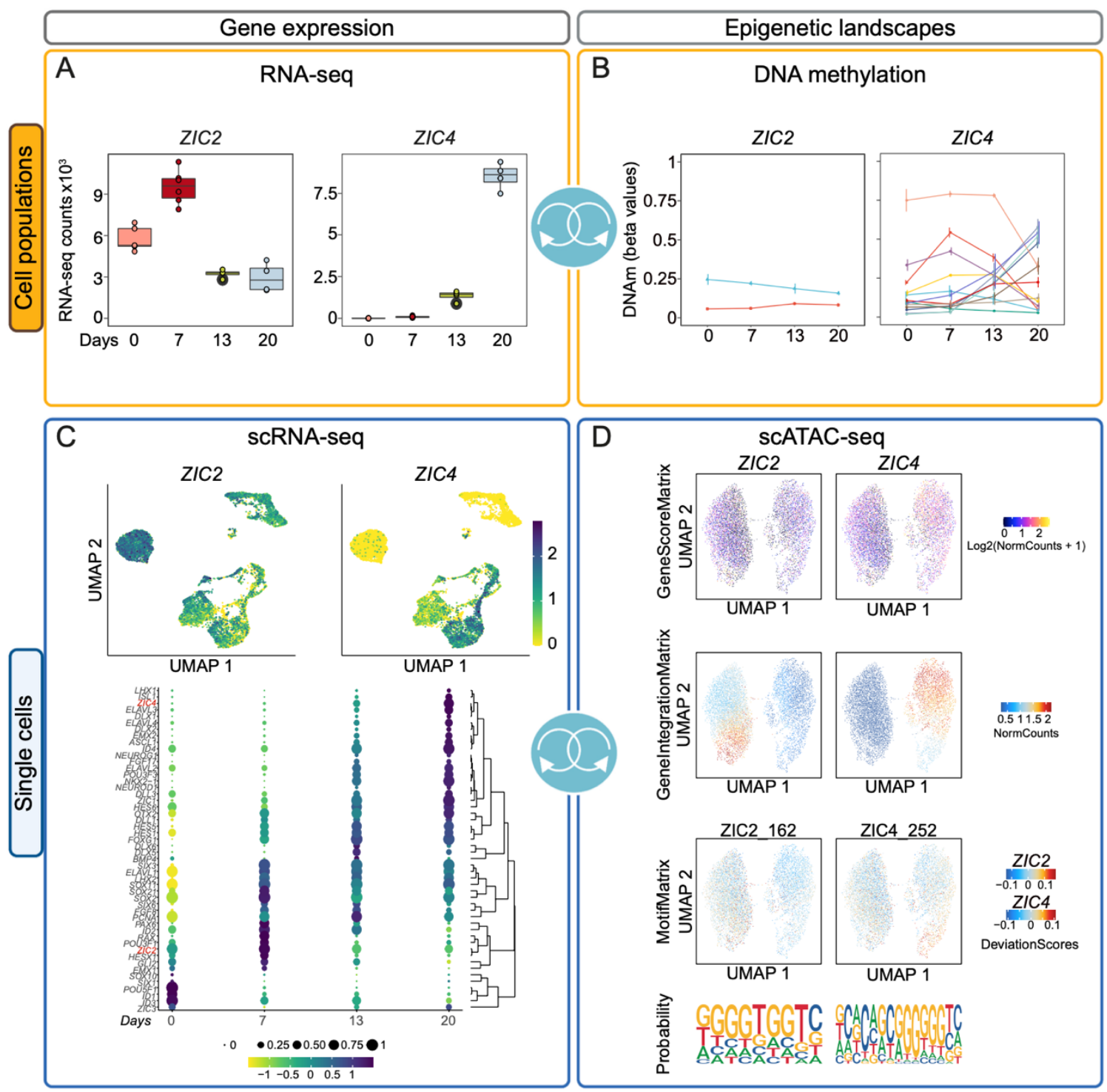

Figure 7. An example of the possibilities and potential applications of the 4D data showcasing the ZIC2 and ZIC4 genes. A) Normalized global gene expression counts for ZIC2 and ZIC4 from Days 0, 7, 13 and 20. B) Significant CpGs in gene loci ZIC2 and ZIC4 (derived from MORE) for Days 0, 7, 13 and 20. DNAm is represented as mean + - standard deviation. C) Representative UMAPs showing cluster specific and differentiation-driven gene expression across all four timepoints for ZIC2 and ZIC4. A bubble plot representing gene expression and hierarchical clustering of TFs highly relevant to neuronal differentiation across Days $0,7,13$ and 20. D). The upper UMAPS represent inferred gene scores of the openness of the ZIC2 and ZIC4 gene loci. In middle UMAPs the gene integration shows correlation of gene expression with chromatin opening of the ZIC2 and ZIC4 gene loci. The lower UMAPs represent motif footprinting demonstrating preferential opening in different cell clusters for ZIC2 and ZIC4 with the representative sequence logos identified in accessible regions in the dataset below. 


\section{Discussion}

Here we present comprehensive multi-omics analyses to characterize a novel neuronal differentiation protocol from pluripotent ESCs towards a ventrally committed, telencephalic population of progenitors, mature and immature neurons. We assessed stage-to-stage transition using ddPCR and immunofluorescence imaging, and used scRNA-seq and bulk RNA-seq to validate cell populations over time. scATAC-seq and DNAm analysis further characterized the epigenetic and gene regulatory landscapes.

The deconvolution of early human neuronal differentiation at the level of molecular regulation provides insight to an otherwise inaccessible developmental window. Animal models are valuable, but evidence shows that the human neocortex develops under the effect of additional mechanism (Massimo and Long, 2021; Pinson and Huttner, 2021; Xing et al., 2021). Thus, neuronal differentiation studies from PSCs provide an alternative method to characterize developmental transcriptome trajectories and the roles of specific genes in human brain formation and patterning.

To specify the patterning and maturation identities of the cells, we followed up the trajectories of major TFs (O'Leary and Sahara, 2008). Ventral telencephalic markers, such as EMX2 and ASCL1, were already expressed at the end of Stage II, while dorsal telencephalic markers such as EMX1 and NEUROG2 were absent. Absence of expression of HOXB2, PAX7 and GBX2 at any timepoint, confirmed that the selfpatterning phase after neural induction, had no effect on lineage commitment and no cells differentiated to hindbrain, midbrain or thalamic lineages.

GO analyses revealed stage dependent enrichment of biological processes correlated to neurogenesis, pattern specification, signalling and neurotransmitter regulation, migration, synaptic organization and neuronal maturation. The DNAm analyses showed alternating, stage-dependent changes for various patterning genes and TFs important to neurogenesis. In most developmentally regulated genes involved in neuronal lineage commitment, DNAm levels decreased upon transcriptional activation and increased for genes becoming repressed during the time of differentiation. We also observed that sometimes downregulation of gene expression in the self-patterning stage might intersect the upregulation of gene expression seen both in stage I of neural induction of hESCs and during maturation at stage III. This could be due to the combined effect of non-CpG and CpG DNAm implicated in the regulation of RNA splicing in ESCs and neurons, respectively (Ball et al., 2009; Laurent et al., 2010). Non-CpG DNAm accumulates in neurons during synaptogenesis and synaptic pruning (Lister et al., 2013), but CpH DNAm is associated with transcriptional repression (Xie et al., 2012). Whether and how $\mathrm{CpH}$ DNAm plays a role in self patterning following the LSX induction is not known, and future studies are needed to explore this. Furthermore, the in vitro model for DNAm changes presented here, is advantageous for neuropharmacological studies. Whether these changes can be translated to distinct early developmental events, cannot be ascertained. The direction of causality of 
epigenetic regulation for early brain development, can however further correlate in vitro models to sets of open cis-regulatory elements and the regulation of TF-centred networks. The identification of common DNAm modification sites and chromatin openness regions may present candidate loci for future studies of early human development and may advance translational studies of the impact of drugs used early in human pregnancy.

The effect of loss of pluripotency towards neuralization, irrespective of the intermediate timepoints, was investigated through an integrative analysis of the ScATAC- and scRNA-seq. We used ArchR (Granja et al., 2021) for this analysis as this pipeline was flexible for small ATAC-seq and scRNA-seq datasets. The juxtaposition of the transcriptome to the regulatory elements in ESC and differentiating Day 20 cells can infer gene regulatory network information. We identified linked sets of genes unique to each state, that may comprehensively profile individual cells. Such approaches are highly informative. They can infer epigenome causality not restricted to these analyses, but also to studies of the effect of drugs in early brain physiology and development.

\section{Strengths of study}

Although numerous studies have used the LSX cocktail for neural induction, to our knowledge this is the first study that has shared all scRNA data in such transparent and interactive format. Thus, a strength of this study is the presentation of our singlecell data in two visualization tools, ShinyCell and inhouse developed ShinyArchR.UiO (Ouyang et al., 2021; Sharma et al., 2021), that are openly available for users. These tools allow the users to explore candidate genes and utilize a comprehensive set of functionalities, beyond the fate specification analysis presented here. Furthermore, these tools enable insight into the molecular and structural partners of stage-specific markers and time-stamped TFs, their transcriptional regulation and cell cluster identities. Programming scripts for data analysis are made available and can be easily customized for further studies and the incorporation of other data. Although the protocol does not generate terminally differentiated neurons of a specific subtype, there are numerous advantages. The protocol is cost-effective at the level of culture coating reagents, vessel size and timed passaging. Moreover, the advantage of $2 \mathrm{D}$ culture, defined cell numbers at all passaging steps, reduces the human errors in reproducibility compared to protocols based on confluency evaluation and cumbersome 3D culture setup. As the protocol was designed for neuropharmacological studies, the daily media changes further diminish the effect of short drug half lives in such studies.

\section{Limitations of study}

This study has several limitations at the level of cell characterization. The membrane electrochemical and electrophysiological maturation properties were not evaluated. Moreover, we did not assess neuropeptide diversity, secretion of neurotransmitters or migration. Protein quantification or intracellular localization of markers and trajectories were also beyond the scope of a multi-omics characterization. A limitation of ScATAC- 
seq is the genome-per-cell coverage and open chromatin regions relevant for the individual cell or cell populations may have been missed.

In conclusion, in this study we describe the generation of a novel neuronal differentiation protocol where we used the unparalleled power of multi-omics to understand early events of anterior neuroectodermal fate specification. We assessed the functional regulation of transcription factors and developmentally regulated genes, from loss of pluripotency towards neuronal differentiation. Integration of scATAC-seq and scRNA-seq provide invaluable insight on the complexity of fate decisions and enabling other researchers to finetune future studies. Finally, the reader has access to the single-cell sequencing data in two searchable, user-friendly webtools to visualize intra- and inter- timepoint and cell cluster regulation, interactively.

\section{Acknowledgements}

We thank Marit Ledsaak and Naima Azouzi for technical assistance. The majority of informatic analysis was performed at saga super computing resources (Project NN9632K) provided by UNINETT Sigma2-the National Infrastructure for High Performance Computing and Data Storage in Norway. The sequencing service was provided by the Norwegian Sequencing Centre (www.sequencing.uio.no), a national technology platform hosted by Oslo University Hospital and the University of Oslo and supported by the "Functional Genomics" and "Infrastructure" programs of the Research Council of Norway and the Southeastern Regional Health Authorities. PharmaTox Strategic Research Initiative was supported by Faculty of Mathematics and Natural Sciences, University of Oslo. We acknowledge funding from Research Council of Norway 262484 (R.E.) and 241117 (R.L.); the Swedish Research Council 2019-01157 (A.S.) and grants from the Swedish Brain FO2019-0087 (A.S.) and the Freemasons Children's House of Stockholm (A.S.). The graphical abstract was generated in Biorender.com.

\section{Author Contributions}

Conceptualization, A.S., K.G., R.L., R.E.; Methodology, A.S., M.S., R.L., S.M., K.G., R.E., A.Sh., M.L., M.F.; Writing - Original Draft, A.S., M.S., A.Sh., M.L., K.G., R.L., R.E.; Writing - Review \& Editing, A.S., M.S. R.L., K.G., R.E., A.Sh., M.L., M.F., G.A.; Software, Formal Analysis, and Visualization, A.Sh., M.S., K.G., P.M.B., R.L., R.E.; Investigation and Validation, A.S., M.F., M.S., M.L., S.M., R.E.; Funding, R.L., A.S., and R.E., Supervision, R.L., K.G., A.S., and R.E. Resources G.A., R.L. and R.E.

\section{Declaration of Interest}

The authors declare no competing interest 


\section{Star methods}

KEY RESOURCES TABLE

\begin{tabular}{|c|c|c|}
\hline REAGENT or RESOURCE & SOURCE & IDENTIFIER \\
\hline \multicolumn{3}{|l|}{ Antibodies } \\
\hline OCT4 (1/100) & Santa Cruz & sc-5279 \\
\hline $\beta 3$-tubulin (1/250) & Santa Cruz & sc-80005 \\
\hline OTX2 (1/40) & R\&D & AF1979 \\
\hline SOX2 (1/200) & Abcam & $a b 79351$ \\
\hline PAX6 (1/350) & Abcam & ab195045 \\
\hline NESTIN (1/200) & Abcam & ab22035 \\
\hline $\begin{array}{l}\text { Donkey Anti-Goat lgG H\&L (Alexa } \\
\text { Fluor@ 555) } \\
(1 / 500)\end{array}$ & Abcam & ab150130 \\
\hline $\begin{array}{l}\text { Donkey Anti-Mouse IgG H\&L (Alexa } \\
\text { Fluor® } 555)(1 / 500)\end{array}$ & Abcam & ab150110 \\
\hline $\begin{array}{l}\text { Alexa Fluor }{ }^{\circledR} 488 \text { AffiniPure Donkey } \\
\text { Anti-Rabbit } \lg G(H+L)(1 / 250)\end{array}$ & $\begin{array}{l}\text { Jackson } \\
\text { ImmunoResearch }\end{array}$ & $711-545-152$ \\
\hline \multicolumn{3}{|c|}{ Chemicals, peptides, and recombinant proteins } \\
\hline $\begin{array}{l}\text { Geltrex }{ }^{\mathrm{TM}} \text { LDEV-Free, hESC-Qualified, } \\
\text { Reduced Growth Factor Basement } \\
\text { Membrane Matrix }\end{array}$ & ThermoFisher & A1413302 \\
\hline KnockOut ${ }^{\mathrm{TM}}$ DMEM & ThermoFisher & 10829018 \\
\hline PBS, no calcium, no magnesium & $\begin{array}{l}\text { ThermoFisher / } \\
\text { GIBCO }\end{array}$ & 14190 \\
\hline Dimethyl-sulfoxide, DMSO & Sigma-Aldrich & D8418 \\
\hline Accutase $^{\mathrm{TM}}$ Cell Detachment Solution & $\begin{array}{l}\text { STEMCELL } \\
\text { Technologies }\end{array}$ & 7920 \\
\hline UltraPure 0.5 M EDTA, pH 8.0 & ThermoFisher & 15575020 \\
\hline RHO/ROCK Pathway Inhibitor Y-27632 & $\begin{array}{l}\text { STEMCELL } \\
\text { Technologies }\end{array}$ & SCM075 \\
\hline
\end{tabular}




\begin{tabular}{|c|c|c|}
\hline Essential $8^{\mathrm{TM}}$ Medium & ThermoFisher & A1517001 \\
\hline Poly-L-ornithine hydrobromide & $\begin{array}{l}\text { Sigma-Aldrich/ } \\
\text { Merck }\end{array}$ & P3655 \\
\hline Fibronectin (Bovine Protein, Plasma) & ThermoFisher & 33010018 \\
\hline N2 supplement (100X) & ThermoFisher & 17502048 \\
\hline Advanced DMEM/F-12 & ThermoFisher & 12634028 \\
\hline GlutaMAX ${ }^{\mathrm{TM}}$ Supplement & $\begin{array}{l}\text { GIBCO/ } \\
\text { ThermoFisher }\end{array}$ & 35050061 \\
\hline Penicillin Streptomycin $(10,000$ U/mL) & ThermoFisher & 15140122 \\
\hline LDN-193189 & $\begin{array}{l}\text { STEMCELL } \\
\text { Technologies }\end{array}$ & 72148 \\
\hline SB 431542 (hydrate) & $\begin{array}{l}\text { Sigma-Aldrich / } \\
\text { Merck }\end{array}$ & S4317 \\
\hline XAV939 & $\begin{array}{l}\text { STEMCELL } \\
\text { Technologies }\end{array}$ & 72674 \\
\hline B-27 ${ }^{\text {TM }}$ Supplement (50X), serum free & ThermoFisher & 17504044 \\
\hline Recombinant Human FGF basic & Peprotech & $100-18 B$ \\
\hline Recombinant Human EGF, Animal-Free & Peprotech & $A F-100-15$ \\
\hline $\begin{array}{l}\text { Invitrogen }^{\mathrm{TM}} \\
\text { ProLong }^{\mathrm{TM}} \\
\text { Gold Antifade } \\
\text { Mountant with } \\
\text { DAPI }\end{array}$ & $\begin{array}{l}\text { Fisher Scientific/ } \\
\text { Invitrogen }\end{array}$ & P36931 \\
\hline Paraformaldehyde & Sigma-Aldrich & 158127 \\
\hline Triton X-100 & ThermoFisher & 11332481001 \\
\hline Tween-20 & Sigma-Aldrich & P1379 \\
\hline Normal-Horse-Serum-Blocking-Solution & $\begin{array}{l}\text { BioNordica/ } \\
\text { Vectorlabs }\end{array}$ & S-2000-20 \\
\hline Bovine Serum Albumin & Sigma-Aldrich & A2153 \\
\hline
\end{tabular}




\begin{tabular}{|c|c|c|}
\hline $\begin{array}{l}\text { Countess }{ }^{\mathrm{TM}} \text { Cell Counting Chamber } \\
\text { Slides }\end{array}$ & ThermoFisher & C10312 \\
\hline RNeasy Mini Kit & Qiagen & 74106 \\
\hline RNAse-Free DNase Set & Qiagen & 79254 \\
\hline RNA/DNA purification kit & Norgen Biotek Corp. & $298-48700$ \\
\hline RNase-Free DNase I Kit & Norgen Biotek Corp. & $298-25720$ \\
\hline Qubit $^{\text {TM }}$ RNA BR Assay Kit & $\begin{array}{l}\text { ThermoFisher/Invitro } \\
\text { gen }\end{array}$ & Q10211 \\
\hline QuantiTect Reverse Transcription Kit & Qiagen & 205311 \\
\hline ddPCR Supermix for Probes (no dUTP) & BioRad & $186-3024$ \\
\hline Droplet Generation Oil for Probes & BioRad & $186-3005$ \\
\hline TruSeq Stranded mRNA Library Prep Kit & Illumina & 20020595 \\
\hline $\begin{array}{l}\text { IDT for Illumina - TruSeq RNA UD } \\
\text { Indexes }\end{array}$ & Illumina & 20022371 \\
\hline $\begin{array}{l}\text { NovaSeq } 6000 \text { S1 Reagent Kit v1.5 (200 } \\
\text { cycles) }\end{array}$ & Illumina & 20028318 \\
\hline $\begin{array}{l}\text { Infinium MethylationEPIC BeadChip Kit } \\
\text { (96 samples) }\end{array}$ & Illumina & WG-317-1003 \\
\hline 30 mm MACS SmartStrainers & Miltenyi Biotech & $130-110-915$ \\
\hline $\begin{array}{l}\text { Chromium Single Cell 3' Library \& Gel } \\
\text { Bead Kit v3 }\end{array}$ & 10x Genomics & 1000075 \\
\hline Chromium i7 Multiplex Kit & 10x Genomics & 120262 \\
\hline $\begin{array}{l}\text { NextSeq 500/550 High Output Kit (150 } \\
\text { Cycles) }\end{array}$ & Illumina & 20024907 \\
\hline Next GEM Chip H Single Cell Kit & 10x Genomics & 1000162 \\
\hline $\begin{array}{l}\text { Next GEM Single Cell ATAC Library \& } \\
\text { Gel Bead Kit v1.1 }\end{array}$ & 10x Genomics & 1000176 \\
\hline Chromium i7 Multiplex Kit N Set A & 10x Genomics & 1000084 \\
\hline $\begin{array}{l}\text { NovaSeq } 6000 \text { SP Reagent Kit (100 } \\
\text { cycles) }\end{array}$ & Illumina & 20028401 \\
\hline \multicolumn{3}{|l|}{ Deposited data } \\
\hline
\end{tabular}




\begin{tabular}{|c|c|c|}
\hline $\begin{array}{l}\text { RNA-seq, DNAm, Infinium Methylation } \\
\text { EPIC, scRNA-seq \& scATAC-seq }\end{array}$ & This paper & $\begin{array}{l}\text { NCBI GEO: } \\
\text { GSE192858 } \\
\text { (Subseries } \\
\text { GSE192854, } \\
\text { GSE192855, } \\
\text { GSE192856, } \\
\text { GSE192857) }\end{array}$ \\
\hline \multicolumn{3}{|c|}{ Experimental models: Human Embryonic Stem Cell lines } \\
\hline Human embryonic cells, HS360 & $\begin{array}{l}\text { Stockholms } \\
\text { Medicinska Biobank } \\
\text { / Sweden }\end{array}$ & HS360 \\
\hline \multicolumn{3}{|l|}{ Oligonucleotides } \\
\hline POU5F1 & $\begin{array}{l}\text { ThermoFisher/TaqM } \\
\text { an }^{\text {TM }}\end{array}$ & Hs00999632_g1 \\
\hline sox2 & $\begin{array}{l}\text { ThermoFisher/TaqM } \\
\text { an }^{\text {TM }}\end{array}$ & Hs01053049_s1 \\
\hline NANOG & $\begin{array}{l}\text { ThermoFisher/TaqM } \\
\text { an }^{\mathrm{TM}}\end{array}$ & Hs04399610_g1 \\
\hline NES & $\begin{array}{l}\text { ThermoFisher/TaqM } \\
\text { an }^{\text {TM }}\end{array}$ & Hs04187831_g1 \\
\hline FOXG1 & $\begin{array}{l}\text { ThermoFisher/TaqM } \\
\text { an }{ }^{\mathrm{TM}}\end{array}$ & Hs01850784_s1 \\
\hline TUBB3 & $\begin{array}{l}\text { ThermoFisher/TaqM } \\
\text { an }^{\text {TM }}\end{array}$ & Hs00801390_s1 \\
\hline MAP2 & $\begin{array}{l}\text { ThermoFisher/TaqM } \\
\text { an }{ }^{\mathrm{TM}}\end{array}$ & Hs00258900_m1 \\
\hline PAX6 & $\begin{array}{l}\text { ThermoFisher/TaqM } \\
\text { an }^{\text {TM }}\end{array}$ & Hs00240871_m1 \\
\hline OTX2 & $\begin{array}{l}\text { ThermoFisher/TaqM } \\
\text { an }{ }^{\mathrm{TM}}\end{array}$ & Hs00222238_m1 \\
\hline VIM & $\begin{array}{l}\text { ThermoFisher/TaqM } \\
\text { an }^{\mathrm{TM}}\end{array}$ & Hs00958111_m1 \\
\hline NEUROD1 & $\begin{array}{l}\text { ThermoFisher/TaqM } \\
\text { an }^{\text {TM }}\end{array}$ & Hs01922995_s1 \\
\hline
\end{tabular}




\begin{tabular}{|c|c|c|}
\hline RPL30 & $\begin{array}{l}\text { ThermoFisher/TaqM } \\
\text { an }^{\text {TM }}\end{array}$ & Hs00265497_m1 \\
\hline $\begin{array}{l}\text { RAF1 } \\
\text { F: tgggaaatagaagccagtgaa } \\
\text { R: cctttaggatctttactgcaacatc }\end{array}$ & Eurofins & $\begin{array}{l}\text { Roche Universal } \\
\text { Probe Library/ } \\
\text { Probe } 56 \\
4688538001\end{array}$ \\
\hline \multicolumn{3}{|l|}{ Equipment } \\
\hline Hot plate & Custom made & $\mathrm{UiO}$ \\
\hline $\begin{array}{l}\text { Microscope: EVOS }{ }^{\circledR} \text { FL Cell Imaging } \\
\text { System }\end{array}$ & ThermoFisher & AMF4300 \\
\hline $\begin{array}{l}\text { Cell counter: Countess }{ }^{\circledR} \text { II FL Automated } \\
\text { Cell Counter }\end{array}$ & ThermoFisher & MQAF1000 \\
\hline $\begin{array}{l}\text { Qubit Fluorometer for nucleic acid } \\
\text { quantification }\end{array}$ & $\begin{array}{l}\text { ThermoFisher / Life } \\
\text { Technologies }\end{array}$ & 2.0 \\
\hline $\begin{array}{l}\text { Bioanalyzer for Nucleic Acid Quality } \\
\text { analysis }\end{array}$ & Agilent & 2100 \\
\hline Nanodrop & ThermoFisher & 2000 \\
\hline $\begin{array}{l}\text { DG8 }{ }^{\mathrm{TM}} \text { Cartridges for } \mathrm{QX} 200^{\mathrm{TM}} / \mathrm{QX} 100^{\mathrm{TM}} \\
\text { Droplet Generator }\end{array}$ & BioRad & $186-4008$ \\
\hline $\begin{array}{l}\text { DG8 }{ }^{\mathrm{TM}} \text { Gaskets for QX200 } \\
\text { Droplet Generator }\end{array}$ & BioRad & $186-3009$ \\
\hline Droplet Generator & BioRad & QX200 \\
\hline Droplet Reader & BioRad & QX200 \\
\hline NovaSeq & Illumina & 6000 \\
\hline NextSeq & Illumina & 500 \\
\hline iScan system & Illumina & \\
\hline 10x Chromium controller & 10x Genomics & \\
\hline \multicolumn{3}{|l|}{ Software } \\
\hline ArchR1.0.1 & & $\begin{array}{l}\text { https://www.archrp } \\
\text { roject.com }\end{array}$ \\
\hline Seurat Version 4 & $\begin{array}{l}\text { (Hao et al., 2021; } \\
\text { Stuart et al., 2019) }\end{array}$ & $\begin{array}{l}\text { https://github.com/ } \\
\text { satijalab/seurat }\end{array}$ \\
\hline
\end{tabular}




\begin{tabular}{|c|c|c|}
\hline Signac & (Stuart et al., 2021) & $\begin{array}{l}\text { https://satijalab.org } \\
\text { /signac/ }\end{array}$ \\
\hline BSgenome1.58.0 & (Pagès, 2020) & $\begin{array}{l}\text { https://rdrr.io/bioc/ } \\
\text { BSgenome/ }\end{array}$ \\
\hline ShinyCell & (Ouyang et al., 2021) & $\begin{array}{l}\text { https://github.com/ } \\
\text { SGDDNB/ShinyCe } \\
\text { II }\end{array}$ \\
\hline ShinyArchR.UiO & (Sharma et al., 2021) & $\begin{array}{l}\text { https://github.com/ } \\
\text { EskelandLab/Shin } \\
\text { yArchRUiO }\end{array}$ \\
\hline CytoTRACE R package (v0.3.3) & (Gulati et al., 2020) & $\begin{array}{l}\text { https://cytotrace.st } \\
\text { anford.edu }\end{array}$ \\
\hline $\begin{array}{l}\text { 10x Genomics Cell Ranger -Count and } \\
\text { 10x Genomics Cell Ranger -Count ATAC }\end{array}$ & 10X genomics & $\begin{array}{l}\text { https://www. } 10 \times g e \\
\text { nomics.com }\end{array}$ \\
\hline $\mathrm{R}$ Programming language & & $\begin{array}{l}\text { https://www.r- } \\
\text { project.org/ }\end{array}$ \\
\hline FIJI & $\begin{array}{l}\text { (Schindelin et al., } \\
\text { 2012) }\end{array}$ & $\begin{array}{l}\text { https://imagej.net/s } \\
\text { oftware/fiji/ }\end{array}$ \\
\hline BSgenome.Hsapiens.UCSC.hg38 & $\begin{array}{l}\text { DOI:10.18129/B9.bio } \\
\text { c.BSgenome.Hsapie } \\
\text { ns.UCSC.hg38 }\end{array}$ & $\begin{array}{l}\text { https://bioconducto } \\
\text { r.org/packages/rel } \\
\text { ease/data/annotati } \\
\text { on/html/BSgenom } \\
\text { e.Hsapiens.UCSC. } \\
\text { hg38.html }\end{array}$ \\
\hline EnsDb.Hsapiens.v86 & $\begin{array}{l}\text { DOI:10.18129/B9.bio } \\
\text { c.EnsDb.Hsapiens.v } \\
86\end{array}$ & $\begin{array}{l}\text { https://bioconducto } \\
\text { r.org/packages/rel } \\
\text { ease/data/annotati } \\
\text { on/html/EnsDb.Hs } \\
\text { apiens.v86.html }\end{array}$ \\
\hline clustree & $\begin{array}{l}\text { (Zappia and } \\
\text { Oshlack, 2018) }\end{array}$ & $\begin{array}{l}\text { https://cran.r- } \\
\text { project.org/web/pa } \\
\text { ckages/clustree/vi } \\
\text { gnettes/clustree.ht } \\
\text { ml\#references }\end{array}$ \\
\hline scater & $\begin{array}{l}\text { (McCarthy et al., } \\
\text { 2017) }\end{array}$ & $\begin{array}{l}\text { https://bioconducto } \\
\text { r.org/packages/rel } \\
\text { ease/bioc/html/sca } \\
\text { ter.html }\end{array}$ \\
\hline
\end{tabular}




\begin{tabular}{|c|c|c|}
\hline DEseq2 & (Love et al., 2014) & $\begin{array}{l}\text { https://bioconducto } \\
\text { r.org/packages/rel } \\
\text { ease/bioc/html/DE } \\
\text { Seq2.html }\end{array}$ \\
\hline GSEA & $\begin{array}{l}\text { (Subramanian et al., } \\
\text { 2005) }\end{array}$ & $\begin{array}{l}\text { https://www.gsea- } \\
\text { msigdb.org/gsea/in } \\
\text { dex.jsp }\end{array}$ \\
\hline Minfi & (Aryee et al., 2014) & $\begin{array}{l}\text { https://www.biocon } \\
\text { ductor.org/packag } \\
\text { es/release/bioc/ht } \\
\text { ml/minfi.html }\end{array}$ \\
\hline limma & (Ritchie et al., 2015) & $\begin{array}{l}\text { https://bioconducto } \\
\text { r.org/packages/rel } \\
\text { ease/bioc/html/lim } \\
\text { ma.html }\end{array}$ \\
\hline missMethyl & $\begin{array}{l}\text { (Phipson et al., } \\
\text { 2016) }\end{array}$ & $\begin{array}{l}\text { https://bioconducto } \\
\text { r.org/packages/rel } \\
\text { ease/bioc/html/mis } \\
\text { sMethyl.html }\end{array}$ \\
\hline MORE & (Conesa, 2018) & $\begin{array}{l}\text { https://github.com// } \\
\text { ConesaLab/MORE }\end{array}$ \\
\hline $\begin{array}{l}\text { Additional tools and more detailed } \\
\text { methods can be found in Supplemental } \\
\text { information and Table S7. }\end{array}$ & This paper & $\begin{array}{l}\text { Custom scripts for } \\
\text { computational } \\
\text { analysis are } \\
\text { available at } \\
\text { https://github.com/ } \\
\text { EskelandLab/scNe } \\
\text { uronaldiff. Single- } \\
\text { cell data can be } \\
\text { explored in } \\
\text { webtools "hESC } \\
\text { Neuronal } \\
\text { Differentiation } \\
\text { scRNA-seq" and } \\
\text { "hESC Neuro } \\
\text { Differentiation } \\
\text { scATAC seq" } \\
\text { at } \\
\text { https://cancell.med } \\
\text { isin.uio.no/ }\end{array}$ \\
\hline
\end{tabular}




\section{RESOURCE AVAILABILITY}

\section{Lead contact}

Further information and requests for resources and reagents should be directed to and will be fulfilled by the lead contact Ragnhild Eskeland (Ragnhild.Eskeland@medisin.uio.no).

\section{EXPERIMENTAL MODEL AND SUBJECT DETAILS}

\section{Human embryonic stem cell (hESC) culture and maintenance and neuronal differentiation protocol}

The full description of the differentiation protocol is described in our protocol manuscript (bioRxiv https://doi.org/10.1101/2022.01.26.477818).

\section{Immunofluorescence analysis}

In brief, cells grown on $13 \mathrm{~mm}$ glass coverslips, were washed once and fixed in $4 \%$ paraformaldehyde for $15 \mathrm{~min}$ at room temperature (RT). After 3 washes, the cells were permeabilized with $0.3 \%$ Triton X-100 (ThermoFisher) in blocking buffer containing $2 \%$ BSA (Sigma-Aldrich) and $0.01 \%$ Tween in $1 \times$ PBS for 30 min at RT, washed 3 times, and blocked with $10 \%$ horse serum for $30 \mathrm{~min}$. Primary antibodies were diluted (as in $\mathrm{KRT}$ ) in $1 \times \mathrm{PBS}$ containing $0.03 \%$ Triton $\mathrm{X}-100$, and coverslips were incubated overnight at $4{ }^{\circ} \mathrm{C}$. Next, coverslips were equilibrated at RT for 2 hours and washed 3 times. The secondary antibodies were diluted (see KRT) in 0.01\% Tween-20 (SigmaAldrich) and $0.1 \%$ horse serum (BioNordica) in $1 \times \mathrm{PBS}$, and coverslips were incubated for one hour at RT. The coverslips were washed 3 times and mounted on microscope slides using the ProLong ${ }^{\text {TM }}$ Gold Antifade Mountant containing DAPI (Fisher Scientific) to counterstain cell nuclei. Washing steps lasted 15 minutes and used $1 \times P B S$. Images were obtained with a DeltaVision high resolution widefield microscope (GE Life Sciences, USA) using the Resolve 3D software and 100X 1.45NA oil objective and processed using the open-source software Fiji (Schindelin et al., 2012).

\section{DNA/RNA isolation}

Genomic DNA and total RNA were isolated by direct lysis in the culture well followed by column-based isolation using RNA/DNA purification kit (Norgen Biotek). The RNase-Free DNase I Kit (Norgen Biotek) was applied for on-column removal of genomic DNA contamination from RNA isolates. Three RNA isolates were processed using RNeasy Mini Kit (Qiagen) followed by DNase-treatment using RNAse-Free DNase Set (Qiagen). All isolations were done according to the manufacturer's instructions. Nucleic acid quantification was performed using Qubit (ThermoFisher Scientific), purity was measured using Nanodrop 2000 (ThermoFisher Scientific), while RNA and DNA integrity was assessed using 2100 Bioanalyzer (Agilent Technologies) and 4200 TapeStation (Agilent Technologies), respectively. 


\section{Droplet Digital RT-PCR and RNA expression analysis}

Reverse transcription of total RNA was performed using QuantiTect Reverse Transcription Kit (Qiagen). Subsequent ddPCR reactions were set up using ddPCR Supermix for Probes (No dUTP) (BioRad) and Taqman assays (ThermoFisher) or Universal Probes (Roche) in combination with target primers (Eurofins) as outlined in KRT/Oligonucleotides. Droplets for droplet PCR amplification were generated using the QX200 Droplet Generator (BioRad). Data acquisition and primary analysis was done using the QX200 Droplet Reader (BioRad) and QuantaSoft software (BioRad). All steps were performed according to the manufacturer's instructions. To calculate the number of target copies per ng RNA input, samples were normalized using RPL30 and RAF1 as normalization genes (Coulter, 2018). Statistical comparisons were performed in $\mathrm{R}$ using t-test in ggpubr package v.0.4.0 (Kassambara, 2020). Results were visualized in $\mathrm{R}$ using the tidyverse package (Wickham et al., 2019).

\section{Global RNA-seq}

The sequencing library was prepared with TruSeq Stranded mRNA Library Prep (Illumina) according to manufacturer's instructions. The 19 libraries were pooled at equimolar concentrations and sequenced on an Illumina NovaSeq 6000 S1 flow cell (Illumina) with $100 \mathrm{bp}$ paired end reads. The quality of sequencing reads was assessed using BBMap (Bushnell, 2014), and adapter sequences and low-quality reads were removed. The sequencing reads were then mapped to the GRCh38.p5 index using HISAT2 (Kim et al., 2015). Mapped paired end reads were counted to protein coding genes using featureCounts (Liao et al., 2014). Differential expression analysis was conducted in $\mathrm{R}$ version 3.5.1 ( $\mathrm{R}$ Core Team, 2019) using SARTools v.1.6.8 (Varet et al., 2016) and the DESeq2 v.1.22.1 (Love et al., 2014), and genes were considered significantly differentially expressed with an FDR $<0.01$. Normalized counts were visualized using the tidyverse package v.1.3.0 (Wickham et al., 2019). The heatmaps were generated using the pheatmap package version 1.0.12 (Kolde, 2019). The Waldtest was used to calculate p-values and Benjamini-Hochberg was used to correct for multiple testing. The gene ontology (GO) analysis of a ranked list of differential expressed genes were performed using GSEA software (Subramanian et al., 2005) looking at biological process (BP) terms.

\section{Illumina EPIC array}

DNA methylation status of 22 samples were assessed using the Infinium MethylationEPIC BeadChip v.1.0_B3 (Illumina). Quality control and pre-processing of the raw data was performed in $\mathrm{R}$ using Minfi v.1.36.0 (Aryee et al., 2014). No samples were removed due to poor quality (detection $p$ values $>0.05$ ). Background correction was performed using NOOB method (Triche et al., 2013) and $\beta$ values (ratio of methylated signal divided by the sum of the methylated and unmethylated signal) were normalized using functional normalization (Fortin et al., 2014). Probes with unreliable measurements (detection $p$ values $>0.01)(n=8,818)$ and cross-reactive probes (Chen et al., 2013) $(n=43,256)$ were then removed, resulting in a final data set consisting of 
814,112 probes and 22 samples. Probes were annotated with Illumina Human Methylation EPIC annotation $1.0 \mathrm{~B} 5$ (hg38). Differential methylation (DM) analysis was performed on the $M$ values (log2 of the $\beta$ values) using the limma package (Ritchie et al., 2015), and $\mathrm{CpGs}$ were considered significantly differentially methylated with an FDR < 0.01. GO analysis was performed using top $10 \%$ DM CpGs (DMCs) as input to GOMETH in the missMethyl package version 1.24.0 (Phipson et al., 2016) for BP terms.

\section{Integration of RNA-seq and DNA methylation data}

Data from matching DNA and RNA samples (extracted from the same wells, $n=16$ ) were subsetted to undergo statistical integration. Multi-Omics Regulation (MORE) (Conesa, 2018) was used to identify CpGs that regulate gene expression by applying Generalized Linear Models: normalized counts for differentially expressed genes (from DEseq2) were used as the response variable, CpG M-values (from Minfi) and experimental covariates (Day) were used as predictors. First, CpGs with low variability were filtered and multicollinearity was reduced by grouping highly correlated CpGs. Variable selection was then performed with Elastic Net regression and stepwise (twoways backward) regression. CpGs were considered to significantly regulate gene expression when the regression coefficient $p$-value was $<0.05$. Significant $C p G$ regulators of gene expression were visualized using the Tidyverse package (Wickham et al., 2019) using beta values $(n=22)$ and normalized counts $(n=19)$ from all samples.

\section{Collection of cells and scRNA-seq}

Cells harvested on Days 0, 7, 13 and 20 were washed twice in wells with 1xPBS and detached using Accutase (STEMCELL Technologies) at $37^{\circ} \mathrm{C}$ for $7 \mathrm{~min}$. Cells were triturated 10-15 times to separate into single cells and transferred to centrifuge tubes containing the appropriate base media with $0.05 \%$ BSA (Sigma-Aldrich). Counts were performed using Countess II FL Cell Counter (ThermoFisher Scientific), cells were centrifuged at $300 \mathrm{x} \mathrm{g}$ for $5 \mathrm{~min}$ and the supernatant was discarded. Cell pellets were then resuspended in base medium containing $0.05 \%$ BSA and cell aggregates were filtered out using MACS SmartStrainers (Miltenyi). The cells were recounted and processed within 1 hour on the 10x Chromium controller (10x Genomics). Approximately 2,300 cells were loaded per channel on the Chromium Chip B (10x Genomics) to give an estimated recovery of 1,400 cells. The Chromium Single Cell 3' Library \& Gel Bead Kit v3 (10x Genomics) and Chromium i7 Multiplex Kit (10x Genomics) were used to generate scRNA-seq libraries, according to the manufacturer's instructions. Libraries from 16 samples were pooled together based on molarity and sequenced on a NextSeq 550 (Illumina) with 28 cycles for read 1, 8 cycles for the 17 index and 91 cycles for read 2. For the second sequencing run, libraries were pooled again based on the number of recovered cells to give a similar number of reads per cell for each sample $(33,000$ - 44,000 reads/cell). 


\section{scRNA-seq data analysis}

The Cell Ranger 3.1.0 Gene Expression pipeline (10x Genomics) was used to demultiplex the raw base-call files and convert them into FASTQ files. The FASTQ files were aligned to the GRCh38-3.0.0 human reference genome, and Cell Ranger count was used with default parameters for computing read counts for Days 0, 7, 13 and 20. The sequenced replicates for each day were aggregated into single datasets using Cell Ranger Aggr command. Duplicates, dead cells and cells with greater than 5 median absolute deviations (MADs) for mitochondrial reads were filtered out (McCarthy et al., 2017). We used scTRANSFORM for normalization to better understand cell to cell heterogeneity after performing cell cycle regression analysis (Hafemeister and Satija, 2019; Tirosh et al., 2016) (for more details, see supplemental information). We used a resolution of 0.55 to cluster cells, obtained by determining the optimum number of clusters (cell grouped together sharing similar expression profiles) in the dataset using the Clustree R package (Zappia and Oshlack, 2018) (Fig. S2B and $\mathrm{C}$ ).

\section{scATAC-seq Library Preparation and Sequencing}

Cells were washed twice with 1xPBS and detached to single cell suspension by application of Accutase (STEMCELL Technologies) at $37^{\circ} \mathrm{C}$ for $7 \mathrm{~min}$. The detached cells were washed with appropriate base media with added $0.04 \%$ BSA (SigmaAldrich) and filtered using MACS SmartStrainers (Miltenyi Biotech) to remove cell aggregates. Nuclei isolation was done according to the 10x Genomics protocol CG000169 (Rev D) using 2 minutes of incubation in lysis buffer diluted to $0.1 \mathrm{x}$ and $0.5 \mathrm{x}$ for Day 0 and Day 20 cells, respectively. We used the Countess II FL Cell Counter (ThermoFisher Scientific) to quantify nuclei and confirm complete lysis and microscopy to confirm high nuclei quality. Nuclei were further processed on the 10x Chromium controller (10x Genomics) using Next GEM Chip H Single Cell Kit (10x Genomics), Next GEM Single Cell ATAC Library \& Gel Bead Kit v1.1 (10 x Genomics) and Chromium i7 Multiplex Kit N Set A (10x Genomics) according to the Next GEM Single Cell ATAC Reagent Kits v1.1 User Guide (CG000209, Rev C). The targeted nuclei recovery was 5,000 nuclei per sample. The resulting 4 sample libraries were sequenced on a NovaSeq Sp flow cell (Illumina) with 50 cycles for read 1, 8 cycles for the i7 index read, 16 cycles for the i5 index read and 49 cycles for read 2.

\section{scATAC sequencing analysis}

Cell Ranger ATAC version 1.2.0 with reference genome GRCh38-1.2.0 was used to pre-process scATAC-seq raw sequencing data into FASTQ files. Single cell accessibility counts for the cells were generated from reads using the 'cellranger-atac count' pipeline. Reference genome HG38 used for alignment and generation of singlecell accessibility counts was obtained from the 10x Genomics (https://support.10xgenomics.com/single-cell-atac/software/downloads/).

Downstream analysis of the scATAC-seq data was performed using the $\mathrm{R}$ package ArchR v1.0.1 (Granja et al., 2021). A tile matrix of 500-bp bins was constructed after 
quality control, removal of low-quality cells and doublet removal using the doubletfinder function of ArchR. The ArchR Project contained the filtered cells that had a TSS enrichment below 3 and $<1000$ fragments. A layered dimensionality reduction approach utilizing Latent Semantic Indexing (LSI) and Singular Value Decomposition (SVD) applied on Genome-wide tile matrix. Uniform Manifold approximation and projection (UMAP) was performed to visualize data in 2D space. Louvain Clustering methods implemented in R package Seurat (Stuart et al., 2019) was used for clustering of the single-cell accessibility profiles.

\section{References}

Al-Naama, N., Mackeh, R., and Kino, T. (2020). C2H2-Type Zinc Finger Proteins in Brain Development, Neurodevelopmental, and Other Neuropsychiatric Disorders: Systematic Literature-Based Analysis. Front Neurol 11, 32.

Arai, Y., Pulvers, J.N., Haffner, C., Schilling, B., Nüsslein, I., Calegari, F., and Huttner, W.B. (2011). Neural stem and progenitor cells shorten S-phase on commitment to neuron production. Nat Commun 2, 154.

Aruga, J., and Millen, K.J. (2018). ZIC1 Function in Normal Cerebellar Development and Human Developmental Pathology. In Zic Family: Evolution, Development and Disease, J. Aruga, ed. (Singapore: Springer), pp. 249-268.

Aryee, M.J., Jaffe, A.E., Corrada-Bravo, H., Ladd-Acosta, C., Feinberg, A.P., Hansen, K.D., and Irizarry, R.A. (2014). Minfi: a flexible and comprehensive Bioconductor package for the analysis of Infinium DNA methylation microarrays. Bioinformatics 30, 1363-1369.

Baek, S., and Lee, I. (2020). Single-cell ATAC sequencing analysis: From data preprocessing to hypothesis generation. Computational and Structural Biotechnology Journal $18,1429-1439$.

Ball, M.P., Li, J.B., Gao, Y., Lee, J.-H., LeProust, E.M., Park, I.-H., Xie, B., Daley, G.Q., and Church, G.M. (2009). Targeted and genome-scale strategies reveal gene-body methylation signatures in human cells. Nat Biotechnol 27, 361-368.

Becker, K.A., Ghule, P.N., Therrien, J.A., Lian, J.B., Stein, J.L., van Wijnen, A.J., and Stein, G.S. (2006). Self-renewal of human embryonic stem cells is supported by a shortened G1 cell cycle phase. J Cell Physiol 209, 883-893.

Beyer, T.A., Weiss, A., Khomchuk, Y., Huang, K., Ogunjimi, A.A., Varelas, X., and Wrana, J.L. (2013). Switch Enhancers Interpret TGF- $\beta$ and Hippo Signaling to Control Cell Fate in Human Embryonic Stem Cells. Cell Reports 5, 1611-1624.

Boward, B., Wu, T., and Dalton, S. (2016). Concise Review: Control of Cell Fate Through Cell Cycle and Pluripotency Networks. STEM CELLS 34, 1427-1436.

Bushnell, B. (2014). BBMap: A Fast, Accurate, Splice-Aware Aligner (Lawrence Berkeley National Lab. (LBNL), Berkeley, CA (United States)).

Cakir, B., Xiang, Y., Tanaka, Y., Kural, M.H., Parent, M., Kang, Y.-J., Chapeton, K., Patterson, B., Yuan, Y., He, C.-S., et al. (2019). Engineering of human brain organoids with a functional vascular-like system. Nat Methods 16, 1169-1175. 
Chavali, V.R.M., Haider, N., Rathi, S., Vrathasha, V., Alapati, T., He, J., Gill, K., Nikonov, R., Duong, T.T., McDougald, D.S., et al. (2020). Dual SMAD inhibition and Wnt inhibition enable efficient and reproducible differentiations of induced pluripotent stem cells into retinal ganglion cells. Sci Rep 10, 11828.

Chen, L., Tong, Q., Chen, X., Jiang, P., Yu, H., Zhao, Q., Sun, L., Liu, C., Gu, B., Zheng, Y., et al. (2021). PHC1 maintains pluripotency by organizing genome-wide chromatin interactions of the Nanog locus. Nat Commun 12, 2829.

Chen, Y., Lemire, M., Choufani, S., Butcher, D.T., Grafodatskaya, D., Zanke, B.W., Gallinger, S., Hudson, T.J., and Weksberg, R. (2013). Discovery of cross-reactive probes and polymorphic $\mathrm{CpGs}$ in the Illumina Infinium HumanMethylation450 microarray. Epigenetics 8, 203-209.

Chiu, W.T., Charney Le, R., Blitz, I.L., Fish, M.B., Li, Y., Biesinger, J., Xie, X., and Cho, K.W.Y. (2014). Genome-wide view of TGF $\beta / F o x h 1$ regulation of the early mesendoderm program. Development 141, 4537-4547.

Chou, S.-J., and Tole, S. (2019). Lhx2, an evolutionarily conserved, multifunctional regulator of forebrain development. Brain Res 1705, 1-14.

Colasante, G., Simonet, J.C., Calogero, R., Crispi, S., Sessa, A., Cho, G., Golden, J.A., and Broccoli, V. (2015). ARX Regulates Cortical Intermediate Progenitor Cell Expansion and Upper Layer Neuron Formation Through Repression of Cdkn1c. Cerebral Cortex 25, 322335.

Conesa, A. (2018). Multi-Omics REgulation by regression models.

Coulter, S.J. (2018). Mitigation of the effect of variability in digital PCR assays through use of duplexed reference assays for normalization. BioTechniques 65, 86-91.

Fedorova, V., Vanova, T., Elrefae, L., Pospisil, J., Petrasova, M., Kolajova, V., Hudacova, Z., Baniariova, J., Barak, M., Peskova, L., et al. (2019). Differentiation of neural rosettes from human pluripotent stem cells in vitro is sequentially regulated on a molecular level and accomplished by the mechanism reminiscent of secondary neurulation. Stem Cell Research 40, 101563.

Fortin, J.-P., Labbe, A., Lemire, M., Zanke, B.W., Hudson, T.J., Fertig, E.J., Greenwood, C.M., and Hansen, K.D. (2014). Functional normalization of 450k methylation array data improves replication in large cancer studies. Genome Biology 15, 503.

Granja, J.M., Corces, M.R., Pierce, S.E., Bagdatli, S.T., Choudhry, H., Chang, H.Y., and Greenleaf, W.J. (2021). ArchR is a scalable software package for integrative single-cell chromatin accessibility analysis. Nature Genetics 53, 403-411.

Gulati, G.S., Sikandar, S.S., Wesche, D.J., Manjunath, A., Bharadwaj, A., Berger, M.J., llagan, F., Kuo, A.H., Hsieh, R.W., Cai, S., et al. (2020). Single-cell transcriptional diversity is a hallmark of developmental potential. Science.

Hafemeister, C., and Satija, R. (2019). Normalization and variance stabilization of single-cell RNA-seq data using regularized negative binomial regression. Genome Biology 20, 296.

Hao, Y., Tang, S., Yuan, Y., Liu, R., and Chen, Q. (2019). Roles of FGF8 subfamily in embryogenesis and oral-maxillofacial diseases (Review). International Journal of Oncology $54,797-806$. 
Hao, Y., Hao, S., Andersen-Nissen, E., Mauck, W.M., Zheng, S., Butler, A., Lee, M.J., Wilk, A.J., Darby, C., Zager, M., et al. (2021). Integrated analysis of multimodal single-cell data. Cell 184, 3573-3587.e29.

Hasenpusch-Theil, K., West, S., Kelman, A., Kozic, Z., Horrocks, S., McMahon, A.P., Price, D.J., Mason, J.O., and Theil, T. (2018). Gli3 controls the onset of cortical neurogenesis by regulating the radial glial cell cycle through Cdk6 expression. Development 145.

Haswell, J.R., Mattioli, K., Gerhardinger, C., Maass, P.G., Foster, D.J., Peinado, P., Wang, X., Medina, P.P., Rinn, J.L., and Slack, F.J. (2021). Genome-wide CRISPR interference screen identifies long non-coding RNA loci required for differentiation and pluripotency. PLoS One 16, e0252848.

Hong, S.-H., Lee, J.-H., Lee, J.B., Ji, J., and Bhatia, M. (2011). ID1 and ID3 represent conserved negative regulators of human embryonic and induced pluripotent stem cell hematopoiesis. Journal of Cell Science 124, 1445-1452.

Hu, Q.-D., Ang, B.-T., Karsak, M., Hu, W.-P., Cui, X.-Y., Duka, T., Takeda, Y., Chia, W., Sankar, N., Ng, Y.-K., et al. (2003). F3/Contactin Acts as a Functional Ligand for Notch during Oligodendrocyte Maturation. Cell 115, 163-175.

lida, H., Furukawa, Y., Teramoto, M., Suzuki, H., Takemoto, T., Uchikawa, M., and Kondoh, $\mathrm{H}$. (2020). Sox2 gene regulation via the D1 enhancer in embryonic neural tube and neural crest by the combined action of SOX2 and ZIC2. Genes to Cells 25, 242-256.

Ikeda, K., Ookawara, S., Sato, S., Ando, Z., Kageyama, R., and Kawakami, K. (2007). Six1 is essential for early neurogenesis in the development of olfactory epithelium. Developmental Biology 311, 53-68.

Kassambara, A. (2020). ggpubr: "ggplot2" Based Publication Ready Plots.

Kim, D., Langmead, B., and Salzberg, S.L. (2015). HISAT: a fast spliced aligner with low memory requirements. Nat Methods 12, 357-360.

Kirkeby, A., Grealish, S., Wolf, D.A., Nelander, J., Wood, J., Lundblad, M., Lindvall, O., and Parmar, M. (2012). Generation of Regionally Specified Neural Progenitors and Functional Neurons from Human Embryonic Stem Cells under Defined Conditions. Cell Reports 1, 703714.

Kolde, R. (2019). pheatmap: Pretty Heatmaps.

Krishnakumar, R., Chen, A.F., Pantovich, M.G., Danial, M., Parchem, R.J., Labosky, P.A., and Blelloch, R. (2016). FOXD3 Regulates Pluripotent Stem Cell Potential by Simultaneously Initiating and Repressing Enhancer Activity. Cell Stem Cell 18, 104-117.

Kurtz, A., Zimmer, A., Schnütgen, F., Brüning, G., Spener, F., and Müller, T. (1994). The expression pattern of a novel gene encoding brain-fatty acid binding protein correlates with neuronal and glial cell development. Development 120, 2637-2649.

Laukoter, S., Beattie, R., Pauler, F.M., Amberg, N., Nakayama, K.I., and Hippenmeyer, S. (2020). Imprinted Cdkn1c genomic locus cell-autonomously promotes cell survival in cerebral cortex development. Nat Commun 11, 195.

Laurent, L., Wong, E., Li, G., Huynh, T., Tsirigos, A., Ong, C.T., Low, H.M., Kin Sung, K.W., Rigoutsos, I., Loring, J., et al. (2010). Dynamic changes in the human methylome during differentiation. Genome Res 20, 320-331. 
Lee, S.-J., Jung, Y.H., Oh, S.Y., Yong, M.S., Ryu, J.M., and Han, H.J. (2014). Netrin-1 Induces MMP-12-Dependent E-Cadherin Degradation Via the Distinct Activation of PKCa and FAK/Fyn in Promoting Mesenchymal Stem Cell Motility. Stem Cells Dev 23, 1870-1882.

Li, X., Sun, L., and Jin, Y. (2008). Identification of karyopherin-alpha 2 as an Oct4 associated protein. Journal of Genetics and Genomics 35, 723-728.

Liao, Y., Smyth, G.K., and Shi, W. (2014). featureCounts: an efficient general purpose program for assigning sequence reads to genomic features. Bioinformatics 30, 923-930.

Lister, R., Mukamel, E.A., Nery, J.R., Urich, M., Puddifoot, C.A., Johnson, N.D., Lucero, J., Huang, Y., Dwork, A.J., Schultz, M.D., et al. (2013). Global epigenomic reconfiguration during mammalian brain development. Science 341, 1237905.

Liu, L., Michowski, W., Kolodziejczyk, A., and Sicinski, P. (2019). The cell cycle in stem cell proliferation, pluripotency and differentiation. Nat Cell Biol 21, 1060-1067.

Love, M.I., Huber, W., and Anders, S. (2014). Moderated estimation of fold change and dispersion for RNA-seq data with DESeq2. Genome Biol. 15, 550.

Main, H., Hedenskog, M., Acharya, G., Hovatta, O., and Lanner, F. (2020). Karolinska Institutet Human Embryonic Stem Cell Bank. Stem Cell Research 45, 101810.

Major, T., Powers, A., and Tabar, V. (2016). Derivation of Telencephalic Oligodendrocyte Progenitors from Human Pluripotent Stem Cells. Current Protocols in Stem Cell Biology 39, 1H.10.1-1H.10.23.

Maksimovic, J., Oshlack, A., and Phipson, B. (2021). Gene set enrichment analysis for genome-wide DNA methylation data. Genome Biology 22, 173.

Maroof, A.M., Keros, S., Tyson, J.A., Ying, S.-W., Ganat, Y.M., Merkle, F.T., Liu, B., Goulburn, A., Stanley, E.G., Elefanty, A.G., et al. (2013). Directed Differentiation and Functional Maturation of Cortical Interneurons from Human Embryonic Stem Cells. Cell Stem Cell 12, 559-572.

Massimo, M., and Long, K.R. (2021). Orchestrating human neocortex development across the scales; from micro to macro. Semin Cell Dev Biol S1084-9521(21)00242-1.

McCarthy, D.J., Campbell, K.R., Lun, A.T.L., and Wills, Q.F. (2017). Scater: pre-processing, quality control, normalization and visualization of single-cell RNA-seq data in R.

Bioinformatics 33, 1179-1186.

Mistri, T.K., Devasia, A.G., Chu, L.T., Ng, W.P., Halbritter, F., Colby, D., Martynoga, B., Tomlinson, S.R., Chambers, I., Robson, P., et al. (2015). Selective influence of Sox2 on POU transcription factor binding in embryonic and neural stem cells. EMBO Reports 16, 11771191.

Nadarajah, B., and Parnavelas, J.G. (2002). Modes of neuronal migration in the developing cerebral cortex. Nat Rev Neurosci 3, 423-432.

Ohashi, M., Korsakova, E., Allen, D., Lee, P., Fu, K., Vargas, B.S., Cinkornpumin, J., Salas, C., Park, J.C., Germanguz, I., et al. (2018). Loss of MECP2 Leads to Activation of P53 and Neuronal Senescence. Stem Cell Reports 10, 1453-1463.

O'Leary, D.D.M., and Sahara, S. (2008). Genetic regulation of arealization of the neocortex. Curr Opin Neurobiol 18, 90-100. 
Ouyang, J.F., Kamaraj, U.S., Cao, E.Y., and Rackham, O.J.L. (2021). ShinyCell: simple and sharable visualization of single-cell gene expression data. Bioinformatics.

Pagès, H. (2020). BSgenome: Software infrastructure for efficient representation of full genomes and their SNPs version 1.58.0 from Bioconductor.

Pang, T., Atefy, R., and Sheen, V. (2008). Malformations of cortical development. Neurologist 14, 181-191.

Pfister, A.S., Tanneberger, K., Schambony, A., and Behrens, J. (2012). Amer2 Protein Is a Novel Negative Regulator of Wnt/ $\beta$-Catenin Signaling Involved in Neuroectodermal Patterning*. Journal of Biological Chemistry 287, 1734-1741.

Phipson, B., Maksimovic, J., and Oshlack, A. (2016). missMethyl: an R package for analyzing data from Illumina's HumanMethylation450 platform. Bioinformatics 32, 286-288.

Pinson, A., and Huttner, W.B. (2021). Neocortex expansion in development and evolutionfrom genes to progenitor cell biology. Curr Opin Cell Biol 73, 9-18.

Piper, M., Barry, G., Harvey, T.J., McLeay, R., Smith, A.G., Harris, L., Mason, S., Stringer, B.W., Day, B.W., Wray, N.R., et al. (2014). NFIB-Mediated Repression of the Epigenetic Factor Ezh2 Regulates Cortical Development. J. Neurosci. 34, 2921-2930.

R Core Team (2019). A Language and Environment for Statistical Computing. R Foundation for Statistical Computing.

Riemens, R.J.M., van den Hove, D.L.A., Esteller, M., and Delgado-Morales, R. (2018). Directing neuronal cell fate in vitro: Achievements and challenges. Progress in Neurobiology 168, 42-68.

Ritchie, M.E., Phipson, B., Wu, D., Hu, Y., Law, C.W., Shi, W., and Smyth, G.K. (2015). limma powers differential expression analyses for RNA-sequencing and microarray studies. Nucleic Acids Res 43, e47.

Schindelin, J., Arganda-Carreras, I., Frise, E., Kaynig, V., Longair, M., Pietzsch, T., Preibisch, S., Rueden, C., Saalfeld, S., Schmid, B., et al. (2012). Fiji: an open-source platform for biological-image analysis. Nat Methods 9, 676-682.

Schlosser, G. (2014). Early embryonic specification of vertebrate cranial placodes. WIREs Developmental Biology 3, 349-363.

Sharma, A., Akshay, A., Rogne, M., and Eskeland, R. (2021). ShinyArchR.UiO: user-friendly, integrative and open-source tool for visualization of single-cell ATAC-seq data using ArchR. Bioinformatics.

Smith, Z.D., and Meissner, A. (2013). DNA methylation: roles in mammalian development. Nat Rev Genet 14, 204-220.

Soufi, A., and Dalton, S. (2016). Cycling through developmental decisions: how cell cycle dynamics control pluripotency, differentiation and reprogramming. Development 143, 43014311.

Spalice, A., Parisi, P., Nicita, F., Pizzardi, G., Del Balzo, F., and lannetti, P. (2009). Neuronal migration disorders: clinical, neuroradiologic and genetics aspects. Acta Paediatrica 98, 421433. 
Stricker, S.H., and Götz, M. (2018). DNA-Methylation: Master or Slave of Neural Fate Decisions? Front Neurosci 12, 5.

Ström, S., Holm, F., Bergström, R., Strömberg, A.-M., and Hovatta, O. (2010). Derivation of 30 human embryonic stem cell lines--improving the quality. In Vitro Cell. Dev. Biol. Anim. 46, 337-344.

Stuart, T., Butler, A., Hoffman, P., Hafemeister, C., Papalexi, E., Mauck, W.M., Hao, Y., Stoeckius, M., Smibert, P., and Satija, R. (2019). Comprehensive Integration of Single-Cell Data. Cell 177, 1888-1902.e21.

Subramanian, A., Tamayo, P., Mootha, V.K., Mukherjee, S., Ebert, B.L., Gillette, M.A., Paulovich, A., Pomeroy, S.L., Golub, T.R., Lander, E.S., et al. (2005). Gene set enrichment analysis: A knowledge-based approach for interpreting genome-wide expression profiles. PNAS 102, 15545-15550.

Sun, J., Yang, J., Miao, X., Loh, H.H., Pei, D., and Zheng, H. (2021). Proteins in DNA methylation and their role in neural stem cell proliferation and differentiation. Cell Regen 10 , 7.

Tang, K., Peng, G., Qiao, Y., Song, L., and Jing, N. (2015). Intrinsic regulations in neural fate commitment. Development, Growth \& Differentiation 57, 109-120.

Tchieu, J., Zimmer, B., Fattahi, F., Amin, S., Zeltner, N., Chen, S., and Studer, L. (2017). A Modular Platform for Differentiation of Human PSCs into All Major Ectodermal Lineages. Cell Stem Cell 21, 399-410.e7.

Tirosh, I., Izar, B., Prakadan, S.M., Wadsworth, M.H., Treacy, D., Trombetta, J.J., Rotem, A., Rodman, C., Lian, C., Murphy, G., et al. (2016). Dissecting the multicellular ecosystem of metastatic melanoma by single-cell RNA-seq. Science 352, 189-196.

Triche, T.J., Weisenberger, D.J., Van Den Berg, D., Laird, P.W., and Siegmund, K.D. (2013). Low-level processing of Illumina Infinium DNA Methylation BeadArrays. Nucleic Acids Res 41, e90.

Varet, H., Brillet-Guéguen, L., Coppée, J.-Y., and Dillies, M.-A. (2016). SARTools: A DESeq2- and EdgeR-Based R Pipeline for Comprehensive Differential Analysis of RNA-Seq Data. PLOS ONE 11, e0157022.

Verrotti, A., Spalice, A., Ursitti, F., Papetti, L., Mariani, R., Castronovo, A., Mastrangelo, M., and lannetti, P. (2010). New trends in neuronal migration disorders. European Journal of Paediatric Neurology 14, 1-12.

Wang, H., Wang, X., Xu, X., Kyba, M., and Cooney, A.J. (2016). Germ Cell Nuclear Factor (GCNF) Represses Oct4 Expression and Globally Modulates Gene Expression in Human Embryonic Stem (hES) Cells. J Biol Chem 291, 8644-8652.

Wang, H., Xiao, Z., Zheng, J., Wu, J., Hu, X.-L., Yang, X., and Shen, Q. (2019a). ZEB1 Represses Neural Differentiation and Cooperates with CTBP2 to Dynamically Regulate Cell Migration during Neocortex Development. Cell Reports 27, 2335-2353.e6.

Wang, Q., Zhang, Y., Wang, M., Song, W.-M., Shen, Q., McKenzie, A., Choi, I., Zhou, X., Pan, P.-Y., Yue, Z., et al. (2019b). The landscape of multiscale transcriptomic networks and key regulators in Parkinson's disease. Nat Commun 10, 5234. 
Wickham, H., Averick, M., Bryan, J., Chang, W., McGowan, L.D., François, R., Grolemund, G., Hayes, A., Henry, L., Hester, J., et al. (2019). Welcome to the Tidyverse. Journal of Open Source Software 4, 1686.

Xie, W., Barr, C.L., Kim, A., Yue, F., Lee, A.Y., Eubanks, J., Dempster, E.L., and Ren, B. (2012). Base-Resolution Analyses of Sequence and Parent-of-Origin Dependent DNA Methylation in the Mouse Genome. Cell 148, 816-831.

Xing, L., Wilsch-Bräuninger, M., and Huttner, W.B. (2021). How neural stem cells contribute to neocortex development. Biochemical Society Transactions 49, 1997-2006.

Yao, B., Christian, K.M., He, C., Jin, P., Ming, G., and Song, H. (2016). Epigenetic mechanisms in neurogenesis. Nat Rev Neurosci 17, 537-549.

Zappia, L., and Oshlack, A. (2018). Clustering trees: a visualization for evaluating clusterings at multiple resolutions. GigaScience 7.

Zhang, S., Bell, E., Zhi, H., Brown, S., Imran, S.A.M., Azuara, V., and Cui, W. (2019). OCT4 and PAX6 determine the dual function of SOX2 in human ESCs as a key pluripotent or neural factor. Stem Cell Research \& Therapy 10, 122.

Zheng, X., Boyer, L., Jin, M., Mertens, J., Kim, Y., Ma, L., Ma, L., Hamm, M., Gage, F.H., and Hunter, T. (2016). Metabolic reprogramming during neuronal differentiation from aerobic glycolysis to neuronal oxidative phosphorylation. Elife 5, e13374.

Zhu, Q., Song, L., Peng, G., Sun, N., Chen, J., Zhang, T., Sheng, N., Tang, W., Qian, C., Qiao, Y., et al. (2014). The transcription factor Pou3f1 promotes neural fate commitment via activation of neural lineage genes and inhibition of external signaling pathways. ELife 3, e02224. 